Pour toute citation : références à rappeler

Salès-Wuillemin, E. (2006). Méthodologie de l'enquête, in : M., Bromberg et A., Trognon (Eds.) Psychologie Sociale 1, Presses Universitaires de France, 45-77.

\title{
Méthodologie de l'enquête
}

Edith Salès-Wuillemin

Professeur de Psychologie

Sociale

Université de Paris 8

Introduction

L'objectif de ce chapitre est de présenter la méthode d'enquête en exposant ses différentes phases, de la conception à la remise du rapport. En préambule, il est souligné que l'enquête occupe une place particulière, à l'interface de l'observation et de l'expérimentation.

L'observation permet de recueillir directement des comportements, sans que cela passe par une verbalisation, même si celle-ci vient souvent (et heureusement) compléter le recueil. Par ailleurs, elle se déroule en milieu naturel, lors d'une activité familière impliquant des objets usuels. Il y a intervention minimale de l'observateur, qui ne cherche ni à provoquer, ni à contrôler la situation. L'expérimentation permet d'analyser les conduites des individus dans une situation totalement provoquée, contrôlée et manipulée, parfois même totalement déconnectée de leur activité ordinaire afin d'observer leurs réactions sans qu'un recours aux scripts habituels de conduite soit possible. L'intervention de l'expérimentateur est maximale et la manipulation systématique des conditions expérimentales autorise des conclusions en termes de causalité.

L'enquête est une «méthode interrogative » (Matalon, 1992). Elle permet de mesurer la perception que les individus ont des objets sociaux. L'enquêteur provoque la situation et met les sujets en situation de verbalisation de leurs points de vue, comportements et connaissances. L'enquêteur se trouve ainsi dans un apparent paradoxe parce qu'il tente d'obtenir des réponses spontanées en réponse à un questionnement planifié.

1. conception générale de l'enquête

L'enquête comporte 5 grandes étapes résumées dans la figure 1, chacune comporte un certain nombre de points à aborder.

\section{Insérer ici Figure 1}

1.1 Détermination du thème et de l'objet de l'étude

L'objet retenu doit avoir un caractère social c'est-à-dire faire l'objet d'enjeux économiques, culturels, professionnels, politiques etc. impliquant des groupes sociaux. La visibilité est éga- 
Pour toute citation : références à rappeler

Salès-Wuillemin, E. (2006). Méthodologie de l'enquête, in : M., Bromberg et A., Trognon (Eds.) Psychologie Sociale 1, Presses Universitaires de France, 45-77.

lement importante, elle est présente lorsque l'objet est le centre de débats contradictoires entre groupes (cf. Moliner, 2001). Ainsi, « les violences urbaines » «les OGM» peuvent pleinement être considérés comme des objets sociaux visibles, alors que «les groupes » « le temps » nécessiteraient des conditions bien précises pour être considérés comme tels (s'il s'agit par exemple $\mathrm{du} \ll$ temps de travail » ou de «l'intégration des groupes minoritaires »).

\subsection{Détermination du cadre théorique : rappel de quelques notions}

L'enquête apprécie la relation que des enquêtés ont avec un objet social. Il est important de préciser quel est le type de relation visé (cherche-t-on à mesurer une attitude, des préjugés/stéréotypes ou une représentation ?) pour que l'entretien et le questionnaire ne se résument pas à une suite de questions désordonnées mais à des outils présentant des garanties de validité (prédictive et interprétative notamment).

\subsubsection{Principales propriétés des attitudes et conséquences sur la mesure}

L'étude des attitudes apparaît en psychologie sociale dans la recherche de Thomas et Znaniecki (1918-1920), sur l'intégration des paysans polonais aux Etats-Unis. Définie comme un état mental s'intercalant entre le sujet et les objets sociaux, elle permet d'expliquer les réactions aux stimulations environnementales. Ce concept connaît plus récemment un regain d'intérêt grâce aux travaux de Fazio sur la force des attitudes, ou de Pratkanis, Eagly et Chaiken sur leur structure, ou encore de Petty et Cacciopo sur les conditions de changement et plus particulièrement les processus de persuasion (cf. Bromberg, 1990 ; Bromberg et Dubois, 1996).

Si l'on se réfère plus précisément à Eagly et Chaiken (1998), il est possible de définir l'attitude à partir de plusieurs caractéristiques. Tout d'abord, elle ne peut être mesurée directement, elle doit donc être appréhendée au travers d'indicateurs ou observables (spontanés ou provoqués) qui en sont des manifestations. Par exemple, l'opinion est un indicateur verbal. Elle peut être suscitée grâce à une série de questions, comme « Etes-vous plutôt favorable ou défavorable aux mesures actuelles dans le domaine de l'emploi des jeunes? », «Que pensez-vous du Contrat Première Embauche? », etc. Le comportement (et l'intention comportementale) est un autre indicateur, il peut être mesuré avec un questionnaire au moyen de questions comme « avez-vous participé à des manifestations contre le CPE ?». L'analyse des réponses donne une indication de l'attitude des sujets vis-à-vis des mesures de lutte contre le chômage prises par le gouvernement. Ensuite, il faut souligner que les attitudes sont présentes dans des réseaux attitudinaux. 
Pour toute citation : références à rappeler

Salès-Wuillemin, E. (2006). Méthodologie de l'enquête, in : M., Bromberg et A., Trognon (Eds.) Psychologie Sociale 1, Presses Universitaires de France, 45-77.

La place d'une attitude au sein du réseau dépend de deux facteurs. Tout d'abord le degré de généralité de l'objet sur lequel elle porte : sont distinguées les attitudes supra-ordonnées (attitude à l'égard de l'écologie par exemple) des attitudes infra-ordonnées (attitude sur la construction de centrales nucléaires, l'utilisation des transports en commun ...). Il est admis que les attitudes supra-ordonnées ont une incidence plus grande sur les comportements et qu'elle sont plus stables (cf. la théorie de la dissonance de Festinger, 1957). Ce caractère vient ensuite se combiner avec le degré de centralité de l'attitude. La centralité renvoie à l'importance que revêt l'objet attitudinal pour le sujet. Les attitudes centrales sont fortement liées aux normes, elles déterminent les comportements et présentent une grande stabilité. En principe, la centralité est indépendante de la généralité. Cependant, les attitudes les plus centrales se trouvent le plus souvent être les plus élevées dans la hiérarchie c'est le cas des attitudes relatives à la société, le travail, la politique, la famille, etc ${ }^{1}$. Au sein du réseau, les attitudes ne présentent que des relations de simple connexité, elles peuvent également avoir des liaisons logiques comme l'inclusion (l'attitude face à l'écologie recouvre celle liée au recyclage des déchets) la causalité ou la covariation (l'attitude face à l'arrêt du service militaire peut impliquer celle sur une armée de métier), etc. Cette organisation en réseaux génère et entretient sa propre cohérence, on peut ainsi observer une formation des attitudes par inférence et pas uniquement à partir d'une expérience directe de l'objet attitudinal. Le raisonnement inférenciel peut être inductif, déductif, analogique, etc. Dans cette optique, une attitude supra-ordonnée positive peut déterminer la valeur de celles qu'elle inclut. La disposition en réseau a également une autre conséquence, apparaît un phénomène de propagation dans le changement des attitudes connexes au sein du réseau. Cette contagion est d'autant plus massive que l'attitude touchée a un haut degré de généralité et/ou de centralité. Heureusement cette réactivité est contrebalancée par l'existence de mécanismes de défense comme «l'exposition sélective» ou les «biais dans le souvenir » comme la distorsion et la «mémorisation sélective. Ces mécanismes permettent de limiter les changements au sein du réseau. Enfin il est possible de distinguer trois composantes dans les attitudes (Allport, 1935 ; Rosenberg et Hovland, 1960). La composante affective correspond aux émotions positives ou négatives que l'individu a à l'égard de l'objet attitudinal, ce qui se traduit par une évaluation de cet objet comme étant bon ou mauvais, intéressant ou inintéressant, etc. La composante cognitive renvoie aux connaissances/croyances de l'individu et à la

\footnotetext{
1 Pour des raisons contextuelles, cette configuration peut néanmoins s'inverser : une attitude infra-ordonnée (comme la construction de centrales nucléaires) peut se trouver subitement, pour un individu, en position centrale parce que par exemple, sa maison d'habitation se trouve dans le périmètre de sécurité d'une future centrale.
} 
Pour toute citation : références à rappeler

Salès-Wuillemin, E. (2006). Méthodologie de l'enquête, in : M., Bromberg et A., Trognon (Eds.) Psychologie Sociale 1, Presses Universitaires de France, 45-77.

crédibilité qu'il leur accorde, ce qui se traduit par des jugements de vérité. La composante conative ou énergétique est relative aux comportements de l'individu et à ses intentions comportementales, ce qui se traduit par des procédures, des règles d'action. Dans la théorie ces trois composantes sont cohérentes. Cependant, l'inférence d'une dimension à partir d'une autre peut s'avérer difficile voire être source d'erreur : de nombreuses études empiriques ont en effet montré qu'un individu peut annoncer qu'il est favorable au tri des ordures ménagères et jeter sans distinction dans ses poubelles, le verre et les piles². De la même manière, une personne peut n'avoir aucun renseignement sur un objet attitudinal et avoir néanmoins une réaction affective vis-à-vis de celui-ci.

L'ensemble de ces caractéristiques a des conséquences directes sur la mesure effectuée. Une mesure d'attitude nécessite une implication des sujets en tant qu'individus pour appréhender leur relation personnelle à l'objet. Le niveau de généralité de l'objet attitudinal doit être pris en compte pour pouvoir déterminer les attitudes infra et supra ordonnées puis les mesurer. Il ne suffit pas de mesurer l'attitude vis-à-vis de l'écologie, il faut également poser des questions sur des objets plus spécifiques. Inversement, s'attacher à des objets particuliers sans prendre en compte le niveau supérieur serait insuffisant. Le degré de centralité de l'attitude doit également être mesuré pour appréhender l'incidence de la position attitudinale sur les conduites des individus interrogés, ou sur la résistance possible face à une intervention à but persuasif. De même, l'environnement de l'attitude au sein du réseau attitudinal devra être évalué grâce à une mesure des attitudes connexes. Enfin, doivent être appréciées les différentes dimensions : la mesure portera donc sur les connaissances, les comportements et les affects vis-à-vis de l'objet.

\subsubsection{Principales caractéristiques des préjugés/stéréotypes et conséquences sur la mesure}

Le préjugé correspond à la dimension affective d'une attitude, l'objet du préjugé est nécessairement un groupe d'individus. Le stéréotype renvoie à la dimension cognitive de l'attitude, il correspond aux croyances sociales que les sujets du groupe source ont du groupe cible. Il se traduit par des traits ou des comportements associés de manière arbitraire. Le préjugé et le stéréotype sont en étroite relation de cohérence : la valence du préjugé (positive ou négative) affecte le contenu du stéréotype associé, celui-ci correspond en outre à une justification du préju-

\footnotetext{
2 Parfois cette apparente incohérence peut trouver des justifications rationalisantes (par exemple, invoquer le risque de perte d'emploi pour les personnels employés au tri) pour atténuer l'écart entre les dimensions affective et conative.
} 
Pour toute citation : références à rappeler

Salès-Wuillemin, E. (2006). Méthodologie de l'enquête, in : M., Bromberg et A., Trognon (Eds.) Psychologie Sociale 1, Presses Universitaires de France, 45-77.

gé (il est sous-tendu par une théorie explicative). La manifestation du préjugé et du stéréotype, la discrimination, correspond à la dimension conative de l'attitude. Elle se traduit par des agissements (cf. Yzerbyt et Schadron, 1996 ; Bourhis et Leyens, 1999).

Les études réalisées montrent que la valence du préjugé, le contenu du stéréotype et les comportements discriminants dépendent de l'appartenance groupale de la cible (endogroupe versus exogroupe). Les stéréotypes qui s'appliquent aux exogroupes sont dénommés exostéréotypes, dans ce cas, la valence du préjugé est en principe négative, et la discrimination l'est également. Le stéréotype qui s'applique à l'endogroupe est un endostéréotype. La valence est en général positive, tout comme la discrimination. Ainsi, les américains voient les français « râleurs » et « irrespectueux des règles ». Alors que les français, renversant la mécanique et la valeur explicative, se perçoivent comme « ayant l'esprit critique » et «débrouillards ». Compte tenu de la dépendance des préjugés, des stéréotypes et de la discrimination, à l'appartenance groupale, leur mesure apparaît d'un grand intérêt. Elle permet en effet d'appréhender les relations intergroupes : symétriques ou asymétriques, et dans ce dernier cas, de révéler la nature de l'asymétrie (cf. Sales-Wuillemin, 2005, 2006).

Ces caractéristiques ont des conséquences sur la mesure effectuée. Le dispositif doit mobiliser l'appartenance des sujets à un groupe pour clairement distinguer l'endostéréotype de l'exostéréotype. Ensuite, il faut mettre en évidence la nature de la partition et les positions de chacun des groupes dans celle-ci. Cela se fait en 3 étapes. Tout d'abord, il faut procéder à la vérification de l'existence réelle d'une partition, les groupes doivent se percevoir comme distincts l'un de l'autre. Ensuite, il faut déterminer la nature des relations, sont-elles symétriques ou asymétriques? dit autrement est-on dans une relation paritaire ou une relation majoritaire/minoritaire. Si tel est le cas, il faudra évaluer, grâce à un travail sociologique et historique approfondi, la nature de l'asymétrie et son origine ainsi que le positionnement respectif de chacun des groupes. Par exemple, s'il s'agit d'une partition de valeur, quel est le groupe valorisé/dévalorisé, s'il s'agit d'une partition en fonction du nombre, quel est le groupe majeur/mineur, etc. (pour toutes ces notions cf. Sales-Wuillemin, 2006).

\subsubsection{Spécificités des représentations sociales et conséquences sur la mesure}

L'étude des représentations sociales a été amorcée en psychologie sociale par la recherche de Moscovici (1961). Jodelet (1989) les définit comme un ensemble de connaissances/croyances correspondant à un système d'interprétation du réel construit conjointement par un groupe afin 
Pour toute citation : références à rappeler

Salès-Wuillemin, E. (2006). Méthodologie de l'enquête, in : M., Bromberg et A., Trognon (Eds.) Psychologie Sociale 1, Presses Universitaires de France, 45-77.

de gérer la réalité sociale. Il est possible de tirer plusieurs conclusions à partir de cette définition. Tout d'abord, ces connaissances ne sont pas scientifiques, mais «de sens commun » ou «naïves ». Ensuite, elles sont partagées par le groupe, elles peuvent ainsi faciliter les communications interindividuelles et limiter les conflits. De plus, elles traduisent le positionnent $d u$ groupe dans un ensemble social. Enfin, elles ont un impact à un niveau individuel (définition de l'identité, modèle de conduites et de pensée, implications affectives) et social (expression des groupes sociaux et transformations sociales).

L'enquête réalisée par Jodelet (1986) sur la représentation sociale de la folie, réalisée auprès d'habitants hébergeant des malades mentaux, permet d'illustrer les caractéristiques du contenu d'une représentation sociale. Elle montre que la cohérence de la représentation est assurée par une "théorie psychiatrique naïve" mettant en jeu deux sources de la maladie : une atteinte du cerveau ou des nerfs ${ }^{3}$. Dans le premier cas, le malade est réputé inoffensif, il est intégré dans la famille d'accueil, dans le second, il est pressenti dangereux, il est écarté. Ce système de catégorisation transparaît au travers de dénominations spécifiques : le malade mental ("Bredin ») est socialement différenciable des non malades ("Civils"). Les « Bredins » se subdivisent en souscatégories (l'«innocent», le «fou mental », etc.), qui permettent de ranger d'emblée tout nouveau venu et d'adapter son comportement.

La structure des représentations sociales a été décrite grâce à la théorie du noyau central (Flament, 1989 ; Abric, 1989) qui prédit que toute représentation s'organise en deux systèmes. Le Système Central (SC) est composé d'éléments qui structurent et orientent la représentation dans son ensemble. Le Système Périphérique (SP) comprend des éléments qui particularisent la représentation, décryptent la réalité et protègent le système central. Dans l'optique structurale, pour dire qu'une représentation se transforme, il faut qu'il y ait modification des éléments du SC. Elle peut avoir plusieurs origines, mais résulte d'une contradiction entre le SC et l'environnement. Le cas le plus typique étant le changement des pratiques du groupe (cf. Guimelli, 1989).

L'ensemble de ces caractéristiques a des conséquences directes sur la mesure. Mesurer une représentation suppose un objet (sur lequel porte la représentation) et un substrat (un groupe qui en est porteur), ce qui implique que les individus soient interrogés en tant que membre d'un groupe précis, c'est cette appartenance catégorielle qu'il leur faudra mobiliser. De plus, le

\footnotetext{
3 Parallèlement, l'explication de la maladie mentale se fait à travers d'expressions particulières "un détraquement des nerfs" dû à "un choc" une "peur de guerre qu'est restée ici" ou encore "sa femme l'a quitté, c'est quelque chose qui est resté là dans le cerveau et qui a tourné".
} 
Pour toute citation : références à rappeler

Salès-Wuillemin, E. (2006). Méthodologie de l'enquête, in : M., Bromberg et A., Trognon (Eds.) Psychologie Sociale 1, Presses Universitaires de France, 45-77.

groupe n'est pas choisi au hasard, il l'est parce qu'il est censé être le plus signifiant en regard de l'objet et de l'ensemble social considéré. Pour montrer sa spécificité il faudra le comparer à d'autres groupes sociaux. L'analyse de la représentation peut également révéler le positionnement de chacun au sein de l'ensemble social. Par exemple, la représentation de l'hygiène diffère selon que l'on est médecin, infirmier, étudiant spécialisé, ou étudiant non spécialisé en soins infirmiers, parce que la relation à l'objet dépend du rôle, des connaissances, des pratiques etc. à propos de cet objet (Sales-Wuillemin, 2005).

1.3 De la formulation de la question d'étude à la formulation d'une problématique

\subsubsection{La question d'étude}

La question d'étude renvoie au questionnement du chercheur en relation à un problème qui se pose, il peut être théorique ou avoir des modalités pratiques. Par exemple, à propos de l'hygiène, la question théorique peut-être «Comment se constitue la représentation d'un objet social comme l'hygiène?» et la question pratique «Comment lutter contre les infections nosocomiales dans les structures de soin?». Bien entendu une déclinaison en une série de questions plus spécifiques peut être envisagée.

\subsubsection{Les objectifs de l'enquête}

Il s'agit à ce stade d'expliciter ce que l'on cherche à faire au travers de l'étude. D'une manière générale, on tend à révéler les liens existant entre des variables prises en compte ou manipulées ${ }^{4}$ et les réponses des enquêtés. Dit autrement entre des Variables Indépendantes (VI) et des Variables Dépendantes (VD). Il peut s'agir par exemple de mettre en évidence le lien qui existe entre la spécialisation des études suivies et la représentation d'un objet comme le Sida, comme nous le développons plus loin.

\subsubsection{Les moyens et contraintes matériels}

Même si l'on est porté par l'enthousiasme d'un projet, les moyens matériels doivent être pris en compte. A-t-on accès à la population visée ? De quel délai bénéficie-t-on ? Les modalités de questionnement sont-elles acceptables? Autant de questions qui sans être centrales en regard

\footnotetext{
${ }^{4}$ Dans l'enquête ces variables peuvent renvoyer à des conditions internes (caractéristiques de la population, comme la formation, les pratiques professionnelles, ...) ou externes (par exemple le type d'habitat...).
} 
Pour toute citation : références à rappeler

Salès-Wuillemin, E. (2006). Méthodologie de l'enquête, in : M., Bromberg et A., Trognon (Eds.) Psychologie Sociale 1, Presses Universitaires de France, 45-77.

de la problématique, peuvent néanmoins avoir pour effet une reformulation radicale de la question d'étude.

\subsubsection{Les règles de formulation d'une problématique}

La problématique est une formulation du problème à résoudre, elle se concrétise par une explication des relations existant entre la question d'étude et le cadre théorique dans lequel s'insère la recherche. Une étude peut ainsi avoir pour objet l'hygiène en milieu hospitalier. L'objectif peut être une analyse des erreurs en ce qui concerne l'application des protocoles d'hygiène ${ }^{5}$. La question d'étude correspondrait à la formulation d'une interrogation sur les causes de ces erreurs. La problématique pourrait préciser que l'approche du problème peut se faire grâce à l'analyse des représentations que les personnels soignants ont de certains protocoles, comme celui du lavage des mains, et de manière plus générale de l'hygiène (cf. Sales-Wuillemin, et al. 2005 ; Dautun, et al. 2005).

\section{La pré-enquête}

Elle permet de situer l'objet d'étude dans un contexte global (sociologique, économique, historique, psychologique) et de formuler des hypothèses générales. Bien que cruciale, cette étape est peu formalisée. Tous les supports ou moyens d'information accessibles sont exploités, il peut ainsi s'agir de réaliser une observation directe ou de mettre en oeuvre une méthode documentaliste.

\subsection{Le choix des variables et leur opérationnalisation}

\subsubsection{La nature des VI dans l'enquête}

L'enquête n'est pas une expérimentation, toutefois pour procéder de manière méthodique on doit distinguer les VI des VD. Cependant, parce que les VI de l'enquête sont spécifiques, il n'est en général pas possible de mettre en évidence des relations causales. Dans l'expérimentation les VI sont en principe provoquées c'est-à-dire manipulées par l'expérimentateur. Chaque VI se décompose en plusieurs modalités que peut prendre la variable. Par exemple, si l'on souhaite montrer l'effet de l'influence majoritaire sur les réponses individuelles, on pourra manipuler les conditions d'énonciation de la réponse en distinguant

\footnotetext{
5 Ces erreurs peuvent être différentes : non application du protocole, application inadaptée, ou application d'un protocole inadéquat.
} 
Pour toute citation : références à rappeler

Salès-Wuillemin, E. (2006). Méthodologie de l'enquête, in : M., Bromberg et A., Trognon (Eds.) Psychologie Sociale 1, Presses Universitaires de France, 45-77.

deux modalités : oral, les sujets entendent les réponses des autres participants et écrit, les réponses des autres ne sont pas communiquées. Les sujets peuvent avoir une tâche de jugement : comparer la longueur d'une ligne étalon à trois autres lignes de différentes longueurs (cf. Asch, 1951). Ce sont ces jugements qui constituent la VD, ils permettent de mesurer l'éventuelle existence de l'effet attendu et son ampleur.

Dans l'enquête, les VI ne sont que très rarement manipulées, elles sont en principe invoquées, cela veut dire qu'elles existent indépendamment de l'action du chercheur. Elles s'apparentent plus à des critères de classification qui permettent de comparer plusieurs situations. Les VI invoquées pouvant être utilisées dans un dispositif d'enquête relatif à l'hygiène hospitalière pourraient être le métier exercé (deux modalités : infirmière versus médecin), l'ancienneté (plus de 15 ans versus de 5 à 10 ans versus moins de 3 ans d'expérience) etc. Les VD correspondent à des indicateurs apparaissant dans les réponses des individus interrogés. Que les VI soient provoquées ou invoquées, la comparaison peut se faire entre plusieurs modalités d'une même VI (comparer les réponses des infirmières et des médecins), ou après croisement des modalités de plusieurs variables (comparer les infirmières ayant plus de 15 ans d'expérience, à celles ayant entre 5 et 10ans ou moins de 3 ans d'expérience). Cependant l'interprétation d'éventuelles différences dépend de ce qui a véritablement été pris en compte au travers du choix des VI et VD. Voici pourquoi ces variables doivent être décrites à un niveau théorique et opérationnel.

\subsubsection{Variable théorique et opérationnelle : pourquoi les distinguer?}

Il est nécessaire de distinguer le phénomène que l'on souhaite faire varier ou simplement prendre en compte (par exemple, l'influence à laquelle peut être soumis un individu, la forme de connaissance qu'il a d'un objet avec lequel il est en relation...) et la façon dont cette variable va se traduire. Si cette distinction n'était pas faite, cela risquerait de conduire à une impasse au moment de l'interprétation. Il est en effet toujours possible d'observer des différences, mais l'interprétation de la signification de cette différence nécessite une connaissance des variables qui en sont à l'origine. Par exemple, une VI théorique «les origine des connaissances acquises à propos d'un objet comme 1'Entreprise $\left.{ }^{6}{ }^{\prime}\right\rangle$ peut comporter 2 modalités que 1'on peut vouloir comparer : une connaissance générale (sans pratique), une connaissance générale doublée d'une pratique. D'un point de vue opérationnel, la première modalité peut se concrétiser au travers du choix d'un ensemble d'individus qui connaissent l'objet entreprise, mais sans jamais

\footnotetext{
6 Comme c'est le cas dans l'étude de Moliner 1993.
} 
Pour toute citation : références à rappeler

Salès-Wuillemin, E. (2006). Méthodologie de l'enquête, in : M., Bromberg et A., Trognon (Eds.) Psychologie Sociale 1, Presses Universitaires de France, 45-77.

avoir eu de contact, sous la forme d'un emploi ou d'un stage (ce qui est le cas de bon nombre d'étudiants en première année d'étude en formation généraliste à l'université) ce groupe pourrait être retenu. Il pourrait être comparé à un autre groupe composé de personnes ayant déjà travaillé en entreprise (des salariés). Cependant pour éviter d'être confronté à une variable confondue, il faudrait contrôler le niveau d'étude. Cela pourrait se faire en retenant un groupe constitué de jeunes diplômés venant récemment d'intégrer leur entreprise, ou un groupe d'étudiant en formation professionnelle ayant déjà réalisé plusieurs stages.

Les VD permettent d'estimer l'effet de la VI. Elles doivent également être distinguées à deux niveaux : un premier dit théorique qui correspond à ce que l'on cherche à mesurer, et un deuxième, dit opérationnel traduisant les indicateurs retenus. Dans le cas présenté, la VD théorique est la représentation sociale. Plusieurs VD opérationnelles (indicateurs) pourraient être retenues, le nombre de mots différents du lexique produits par les individus des deux groupes dans une tâche d'associations verbales à propos de l'objet de représentation, les traits sémantiques portés par ces mots, les relations que les individus entrevoient entre ces mots. Cette mesure pourrait par exemple être réalisée grâce à un questionnaire des schèmes cognitifs de base (SCB), élaboré par Guimelli et Rouquette (1992)7.

\subsection{Règles pour la formulation des hypothèses}

Les hypothèses sont des affirmations provisoires qui seront vérifiées grâce à une mise à l'épreuve. Ceci implique naturellement qu'elles soient exhaustives, pertinentes et vérifiables. L'exhaustivité est en lien avec les VI (invoquées ou provoquées). Une hypothèse par variable peut être formulée pour prédire l'effet simple. Si plusieurs VI sont considérées, il faudra considérer les effets d'interaction. Dans ce dernier cas, une prédiction pour chacun des croisements obtenus peut, sans que ce soit une obligation, être réalisée. L'évaluation de la pertinence d'une hypothèse est plus délicate, elle réside dans le lien existant entre l'effet prédit et le cadre théorique. Pour être pertinente l'hypothèse doit pouvoir découler directement de ce cadre. Les hypothèses doivent être énoncées de manière à pouvoir clairement être confirmées ou infirmées. La vérifiabilité d'une hypothèse repose sur les VD retenues et plus exactement sur les indicateurs qui font l'objet de la mesure. Les hypothèses doivent inclure une référence explicite à ces

\footnotetext{
${ }^{7}$ La méthode des SCB consiste après une tâche d'associations verbales à demander aux sujets d'identifier les liens existant entre le mot inducteur (référant à l'objet de la représentation) et les mots induits (produits par les sujets). Ces liens sont établis grâce à des connecteurs regroupés en 3 métaschèmes : Description, Evaluation et Praxéologie.
} 
Pour toute citation : références à rappeler

Salès-Wuillemin, E. (2006). Méthodologie de l'enquête, in : M., Bromberg et A., Trognon (Eds.) Psychologie Sociale 1, Presses Universitaires de France, 45-77.

indicateurs et prédire les variations attendues. Voici pourquoi, elles sont construites en deux parties. La première, appelée hypothèse générale ou théorique, contient une prédiction de portée générale. La seconde appelée hypothèse spécifique ou opérationnelle contient des indicateurs qui permettront de vérifier la confirmation ou l'infirmation de l'hypothèse. Voici un exemple d'hypothèse :

«La représentation de l'hygiène dépend du niveau de connaissance et de pratique des individus interrogés. On s'attend à ce que la représentation des personnels soignants en activité dans des services de soin à l'hôpital soit différente de celle du grand public.

La représentation des professionnels devrait s'articuler autour d'éléments relatifs à l'hygiène hospitalière (protocoles, asepsie, ...), celle du grand public autour de l'hygiène quotidienne (savon, javel, produit de nettoyage ...)».

2.3 Définition de l'univers de l'enquête et modes de sélection de l'échantillon

\subsubsection{Définition de l'univers de l'enquête}

L'univers de l'enquête fait référence à la population visée en regard des objectifs de l'étude. C'est dans cet univers que sera découpé l'échantillon. Forme de connaissance de l'objet, âge, sexe ... sont autant de critères qui peuvent être retenus. Il est donc indispensable d'introduire des questions d'identification: elles peuvent être directement en lien avec les critères d'inclusion des enquêtés (VI ou VC) ou insérées pour caractériser la population. Elles permettent ensuite d'opérer des tris croisés (exemple : croiser le sexe et l'âge) et montrer le lien avec les réponses attitudinales par exemple.

\subsubsection{Les méthodes d'échantillonnage}

Il existe 5 principales méthodes d'échantillonnage. Le sondage aléatoire est le plus élémentaire, mais pas forcément le plus simple. Il s'agit de tirer au sort un ensemble d'individus dans la population ciblée. Cette technique suppose d'en avoir la liste complète. Pour éviter un biais dans le choix (effet de primauté/récence) il est recommandé d'utiliser une table des nombres au hasard après avoir numéroté tous les individus de la liste. Le sondage aréolaire procède à partir d'un quadrillage du lieu dans lequel se trouve la population ciblée (carte géographique ou schémas des services d'une entreprise par exemple), puis par numérotation des zones, enfin par tirage au sort de certaines aires (d'où le nom), toutes les personnes des zones concernées seront interrogées. Pour ce qui concerne le sondage par strates (ou grappes), chaque strate résulte d'un découpage de la population en regard d'un critère (comme le niveau de revenus, le type d'habitat, la profession...). Un nombre constant d'individus est tiré au sort à l'intérieur de cha- 
Pour toute citation : références à rappeler

Salès-Wuillemin, E. (2006). Méthodologie de l'enquête, in : M., Bromberg et A., Trognon (Eds.) Psychologie Sociale 1, Presses Universitaires de France, 45-77.

cune des strates. Le sondage par quotas est plus raffiné. Il s'agit de reproduire les mêmes caractéristiques entre l'échantillon et la population parente en conservant le même pourcentage de femmes/d'hommes, de haut/moyen/faible niveau de revenu etc. que dans la population parente. Le sondage par échantillon maître repose sur un échantillon «prêt à l'emploi » en tous points conformes à la population parente. Les instituts de sondage en possèdent et les réactualisent régulièrement ${ }^{8}$.

\subsection{Création d'un plan de recueil de données}

Le plan schématise les différentes situations à comparer en relation directe avec les hypothèses et les VI. Il peut contenir une VI avec ses différentes modalités, ou plusieurs VI dont les modalités sont croisées, quand le croisement est systématique -que toutes les modalités sont testées- il s'agit d'un plan factoriel. Il s'agit d'indiquer le nombre de conditions obtenues au terme du croisement et le nombre d'individus interrogés dans chaque condition. Imaginons une étude qui porte sur la représentation des malades atteints du Sida dans laquelle seraient analysés les liens entre le contexte d'acquisition des connaissances (VI théorique à deux modalités : dans le domaine de la santé/du social ${ }^{9}$ ) et la représentation (VD théorique). Pour ce qui concerne la VI opérationnelle nous pourrions comparer deux groupes : des élèves infirmières et assistantes sociales. Le niveau d'étude serait ainsi contrôlé (VC) en le laissant à valeur constante. Imaginons maintenant que cette étude prévoit d'inclure une deuxième VI théorique qui serait la valorisation de la cible, deux modalités : cible valorisée/dévalorisée ${ }^{10}$. Pour ce qui concerne l'opérationnalisation, nous pourrions opter pour la comparaison de deux cibles : des malades atteints du Sida Toxicomanes-Prostitués / malades atteints du Sida Hémophiles-Transfusés ${ }^{11}$. Le plan de recueil comprendrait 4 cases correspondant au croisement de ces deux VI à deux modalités.

\section{Insérer ici Tableau 1}

\footnotetext{
8 On peut toutefois douter de la spontanéité des réponses des répondants pratiquant depuis de nombreux mois cette activité.

${ }^{9}$ L'étude présentée par Morin (1999) semble montrer qu'il existe une différence entre les représentations de ces deux groupes.

10 En référence à l'étude de Apostolidis et Cordival citée dans l'ouvrage coordonné par Gosling en 1996.

11 Si l'on en croit l'étude réalisée par Apostolidis et Cordival (1996) le mode de contraction de la maladie a une incidence sur l'image des malades atteints du Sida, les malades homosexuels et toxicomanes semblent être perçus comme des coupables, alors que les malades transfusés et les hémophiles sont perçus comme des victimes innocentes.
} 
Pour toute citation : références à rappeler

Salès-Wuillemin, E. (2006). Méthodologie de l'enquête, in : M., Bromberg et A., Trognon (Eds.) Psychologie Sociale 1, Presses Universitaires de France, 45-77.

Il est à noter toutefois que la première VI opposerait deux groupes indépendants (élèves infirmières/assistantes sociales) alors que la deuxième pourrait, sans que ce soit une obligation, être à mesure répétée avec des groupes appareillés. En d'autres termes, les mêmes sujets répondraient aux questions relatives aux deux cibles. Dans ce dernier cas, il suffirait d'interroger 100 sujets (au lieu de 200 si la mesure intégrait des groupes indépendants).

3. Première phase de l'enquête : les entretiens

3.1 Utilisation générale de l'entretien de recherche au sein d'une enquête

L'entretien est utilisé avec des objectifs très divers (diagnostic clinique, recrutement, enquête ...). Ce qui donne lieu à une déclinaison importante de pratiques et entraîne parfois un certain nombre de confusions. Il s'agit ici de présenter les spécificités de l'entretien d'enquête au sein des entretiens de recherche. Il peut être utilisé préalablement à la réalisation d'un questionnaire (entretiens exploratoires) ou en lieu et place d'un questionnaire (entretiens d'observation). L'entretien exploratoire permet de se familiariser avec l'image que la population ciblée a de l'objet de l'enquête, de poser des hypothèses spécifiques, de répertorier les réactions des individus à propos de l'objet et de les insérer au sein du questionnaire avec une formulation adaptée (sous la forme de questions à choix multiples par exemple). L'entretien d'observation est utilisé pour révéler directement l'image que la population interviewée a de l'objet de l'enquête ${ }^{12}$. Il est privilégié lorsqu'il s'agit d'appréhender des processus de pensée avec une plus grande finesse, ou de mettre au jour les opérations mentales mises en oeuvre par les individus dans la gestion de la réalité sociale, les attributions causales, la dissonance, l'intériorisation des normes, l'élaboration des attitudes etc. ou encore pour permettre à l'interviewé de traduire sa pensée de manière progressive, modulée et personnelle sans l'enfermer dans un cadre de référence, des schèmes de pensée préétablis. La part d'initiative varie toutefois selon le type d'entretien utilisé et plus particulièrement le mode de conduction.

\subsection{L'entretien de recherche: un contrat de communication particulier}

Le contrat de communication (Ghiglione et al. 1986 ; Blanchet et al. 1987 ; Blanchet, 1991) qui s'applique à l'entretien le fait grandement différer d'une conversation banale où chacun prend la parole en faisant en sorte d'apporter une contribution suffisante, pertinente, claire, etc. de

\footnotetext{
${ }^{12}$ Sans viser la représentativité, la taille de l'échantillon interrogé est plus importante que dans les entretiens exploratoires.
} 
Pour toute citation : références à rappeler

Salès-Wuillemin, E. (2006). Méthodologie de l'enquête, in : M., Bromberg et A., Trognon (Eds.) Psychologie Sociale 1, Presses Universitaires de France, 45-77.

manière à faire progresser la conversation qui se déroule (cf. maximes conversationnelles décrites par Grice, 1975). Tout d'abord, dans l'entretien, les protagonistes poursuivent des buts et ont des enjeux spécifiques. L'interviewer $\left(\mathrm{I}^{\mathrm{er}}\right)$ essaie d'obtenir le maximum d'informations sur un thème donné. Il a des attentes précises, aussi il peut être pris dans une logique économique et mettre tout en œuvre pour que cette quête soit fructueuse sans perte de temps inutile avec des entretiens qui ne répondraient pas au cadre strict de l'étude. De façon en apparence contradictoire, il cherche également à obtenir des informations authentiques, à atteindre une dimension personnelle, fruit d'une implication de l' $I^{\mathrm{e}}$. Voilà pourquoi, pour l' $\mathrm{I}^{\mathrm{er}}$ les enjeux sont forts, chaque entretien doit être un succès. En ce qui concerne $1^{\prime} I^{\text {é }}$, les enjeux sont de tout autre nature. Il est bien souvent pris entre deux exigences contradictoires : le désir d'être agréable et de se plier au jeu de l'entretien, mais également le souci de ne pas dire de choses trop personnelles ou impliquantes qui pourraient mettre en péril son image ${ }^{13}$. Ensuite, la relation $\mathrm{I}^{\mathrm{er}} / \mathrm{I}^{\mathrm{e}}$ apparaît comme une relation faussement symétrique. L' $\mathrm{I}^{\mathrm{e}}$ est à la fois en situation de possession des informations que cherche $1^{\text {'er }}{ }^{\mathrm{er}}$, ce qui le met en quelque sorte en position de force. Mais il est également en position de faiblesse vis-à-vis de $1^{\prime} \mathrm{I}^{\mathrm{er}}$ à qui il attribue une bonne technique et une grande expérience dans la conduite d'entretiens. En se référant à Blanchet et Gottman (1992), il est possible de dire que $1^{\prime} \mathrm{I}^{\mathrm{e}}$ est en position de supériorité vis-à-vis du contenu informationnel alors que $1^{\prime} I^{\text {er }} l^{\prime}$ est du point de vue relationnel. De même, si l'on se réfère à Goffman (1959) l'entretien peut apparaître comme un «jeu de face » particulier, parce que l' ${ }^{\mathrm{er}}$ est « autorisé » à réaliser des ingérences dans le territoire de l' $\mathrm{I}^{\mathrm{e}}$, grâce au pouvoir de questionner. L' ' $\mathrm{e}^{\mathrm{e}}$ risque, de par l'acceptation de ce contrat de communication, de perdre la face, par exemple, s'il doit avouer son ignorance de certaines informations. Enfin, un jeu conversationnel peut également se mettre en place durant l'entretien. L' $\mathrm{I}^{\mathrm{er}}$ a des attentes précises, mais souhaite que l' $\mathrm{I}^{\mathrm{e}}$ s'exprime librement. Ce dernier cherche à répondre librement tout en ayant présentes à l'esprit les attentes supposées de $1^{\prime} \mathrm{I}^{\mathrm{er}}$. Lorsque ces attentes sont trop explicites, $1^{\prime} \mathrm{I}^{\mathrm{e}}$ peut être tenté de les satisfaire (biais de complaisance). Si ces attentes ne sont pas assez explicites, il y a risque d'incertitude, l' $\mathrm{I}^{\mathrm{e}}$ peut alors mettre en œuvre des stratégies de protection et de quête des réactions de $1^{\prime} \mathrm{I}^{\mathrm{er}} 14$.

\subsection{Préparation et passation des entretiens selon leur mode de conduction}

\footnotetext{
13 cf. Bézille, in Blanchet 1985

${ }^{14}$ Selon Bezille, 1985, certains sujets procèdent par essai/erreur, ils changent plusieurs fois de direction pour être sûrs d'avoir pris au moins une fois la bonne.
} 
Pour toute citation : références à rappeler

Salès-Wuillemin, E. (2006). Méthodologie de l'enquête, in : M., Bromberg et A., Trognon (Eds.) Psychologie Sociale 1, Presses Universitaires de France, 45-77.

L'entretien Non Directif (appelé libre, ou en profondeur) s'apparente à la méthode clinique. L'entretien Semi-directif est semi structuré ou centré, il donne un cadre souple à l'I $I^{\text {é }}$ L'entretien Directif est standardisé, il s'apparente au questionnaire. Quelle que soit sa forme, l'entretien est nécessairement introduit par une consigne. On en distingue deux : la consigne de prise de rendez-vous (ou de contact) et la consigne inductrice. La première fixe les enjeux globaux de la situation. Les interlocuteurs doivent en effet être réunis par un enjeu commun, sinon il ne pourra y avoir de communication (« sans enjeu pas de jeu », Ghiglione, et al.1986). De façon pragmatique, il s'agit d'obtenir un accord, donc un engagement de l'interlocuteur concernant sa participation à l'étude. Il ne peut être obtenu sans un apport minimum d'informations sur la recherche effectuée (but de l'étude, thème général, date, lieu, heure, durée approximative et ce tout en restant large et ambigu afin que l'Ié ne puisse pas se préparer et mobiliser des connaissances, voire même faire une recherche pour les compléter (biais de complaisance, biais de conformisme, biais d'estime de soi ...). La consigne inductrice fixe ou rappelle les paramètres $\mathrm{du}$ contrat de communication entre $1^{\mathrm{e}} \mathrm{I}^{\mathrm{er}}$ et $\mathrm{l}^{\mathrm{I}} \mathrm{I}^{\mathrm{e}}$ (statuts, buts de la communication, attentes $\mathrm{I}^{\mathrm{er}}$, ...). Elle est construite, rédigée, apprise par cœur puis récitée lors de chaque entretien afin de les rendre comparables (standardisation de la consigne). La formulation se fait avec beaucoup de soin dans le choix des termes qui déterminent le sens à attribuer au discours ultérieur du sujet et dans la clarté des mots utilisés de manière à ce qu'il n'y ait pas d'ambiguïté sur le thème à traiter. Voilà pourquoi il est conseillé de la pré-tester. Cette consigne doit comporter au moins 5 points. Tout d'abord il faut faire l'annonce du thème, des objectifs généraux de l'entretien, ensuite fixer le statut de l' $I^{e r}$ en rapport avec le cadre dans lequel est réalisé le recueil de données, ce qui permet d'installer la relation. Puis, la durée prévue de l'entretien doit être notifiée de façon à permettre à l' $I^{e}$ de programmer son discours et de s'investir dans l'entretien. Cette durée ne doit pas être inférieure à 20 minutes car au départ l' ${ }^{\prime}$ se réfugie derrière des stéréotypes, même si en réalité il n'y adhère pas. Il se protège, par peur d'un jugement négatif et de l'utilisation qui pourra être faite de ses propos. À la suite, la déclaration de l'enregistrement de l'entretien doit être réalisée, elle se fait dans le respect des règles déontologiques. Elle peut être justifiée auprès de $1^{\prime} \mathrm{I}^{\mathrm{e}}$ en avançant un allègement de la prise de note ainsi qu'une écoute plus attentive. Enfin, la présentation de la question inductrice, sert d'amorce (au sens cognitif du terme), elle a un effet sur les éléments de connaissance, les valeurs, les réactions comportementales, mobilisés par les individus interrogés ${ }^{15}$. De multiples recherches (cf. Sales-

15 Sales-Wuillemin et al. 2003, montrent que si l'on fait varier de manière systématique le terme utilisé pour dési- 
Pour toute citation : références à rappeler

Salès-Wuillemin, E. (2006). Méthodologie de l'enquête, in : M., Bromberg et A., Trognon (Eds.) Psychologie Sociale 1, Presses Universitaires de France, 45-77.

Wuillemin, 2005) montrent que le choix du verbe inducteur est déterminant. C'est lui en effet qui fixe le rapport à l'objet et donc les dimensions qui seront mises en saillance ${ }^{16}$ par les sujets et plus généralement leur style discursif. Voici un exemple de consigne inductrice.

«J'aimerais savoir comment vous expliquez (analysez, examinez, comprenez ...) votre situation par
rapport à votre logement» entraîne un discours explicatif de type réalité à construire (cf. Ghi-
glione, 1988). Cette consigne vise à mettre en évidence la façon dont un sujet comprend un objet
donné. La relation à l'objet est cognitive.
«J'aimerais savoir ce que vous pensez de (comment vous évaluez, jugez ...) votre situation par
rapport à votre logement » oriente vers un discours évaluatif de type réalité comme univers pos-
sible (Ghiglione, 1988). Cette consigne vise en effet à obtenir une évaluation, une « opinion » du
sujet à l'égard d'un objet donné. La relation à l'objet est affective.
«J'aimerais savoir comment vous décririez (retraceriez, raconteriez, énonceriez ...) votre situation
par rapport à votre logement » conduit à un discours narratif de type réalité à affirmer (Ghiglione,
1988). Cette consigne vise en effet à obtenir des données factuelles concernant le sujet ou l'objet.
La relation à l'objet est conative.

Une fois la consigne énoncée, l'entretien peut se dérouler. Pour faciliter la production discursive de 1 ' $\mathrm{I}^{\mathrm{e}}$, l' $\mathrm{I}^{\mathrm{er}}$ produit des marques d'accord et d'attention. Elles peuvent être non verbales (regards, sourires, ...), ou verbales (validations explicites comme « oui » « d'accord » «mmh » etc). Toutes ces marques constituent un feed-back (Wiener, 1948) qui peut remplir une ou plusieurs fonctions.

La notion de Feed-back (traduit sous le terme de rétroaction) désigne à la fois la réaction en retour du récepteur face au message émis par l'émetteur et la communication de cette réaction. Le feed-back peut se présenter sous différentes formes et appartenir à plusieurs systèmes de signes (Bouvron et al. 1986; Argentin et al. 1986) : verbal (émission d'une validation par exemple), paraverbal (production d'une interjection avec un effet intonatif), non verbal (mimiques), spatial (postures). Le Feed-back a trois fonctions essentielles :

1-une fonction de régulation : c'est la fonction minimale, de par sa réaction, l'interlocuteur indique qu'il a perçu la contribution de son partenaire et que l'émission peut se poursuivre normalement. En l'absence de feed-back le partenaire peut s'interroger sur la bonne réception de son intervention.

2-une fonction de cumulation cyclique : c'est une fonction qui peut se surajouter à la première, sans que cela soit obligatoire. Le feed-back peut apporter des éléments d'information nouveaux. Grâce à cette fonction, l'interlocuteur peut contribuer pleinement à l'avancée de la conversation.

3- une fonction de cumulation didactique : c'est une fonction qui peut également se surajouter à la première. Le feed-back peut permettre à l'interlocuteur de signaler ce qu'il a exactement compris, par conséquent le partenaire peut ajuster sa contribution en vue d'une amélioration (de sa clarté, de sa précision, de sa pertinence.....).

gner l'objet de la représentation, les éléments activés par les sujets varient également.

16 Les consignes de type « j'aimerais savoir ce que représente pour vous .....» ne centrent pas sur une dimension particulière de l'objet. 
Pour toute citation : références à rappeler

Salès-Wuillemin, E. (2006). Méthodologie de l'enquête, in : M., Bromberg et A., Trognon (Eds.) Psychologie Sociale 1, Presses Universitaires de France, 45-77.

Les marques d'accord produites par l' $^{\text {er }}$ constituent un feed-back partiel, seule la fonction de régulation est remplie, elles ne peuvent donc suffire au bon déroulement de l'échange, l' $\mathrm{I}^{\text {é a be- }}$ soin d'un retour plus spécifique, sinon son discours devient hésitant et finit par s'arrêter. Aussi l'I $^{\text {er }}$ est-il conduit à produire des relances, c'est-à-dire des reprises plus ou moins libres du discours de $1^{\prime} \mathrm{I}^{\mathrm{e}}$. Toutefois, pour limiter les phénomènes d'influence, elles sont construites de façon à ne remplir que la première et la dernière fonction du feed-back. L'I ${ }^{\text {er }}$ n'apporte pas d'élément informatif, il se contente de renvoyer le discours pour signaler qu'il est à l'écoute (fonction de régulation) et pour conduire $1^{\prime} \mathrm{I}^{\mathrm{e}}$ à expliciter ses propos (fonction de cumulation didactique).

Une relance est formulée par 1 ' ${ }^{\mathrm{er}}$ uniquement lorsque se produit une rupture dans le discours de l'I'́ . En s'inspirant de Blanchet et al. (1992) ainsi que de Chauchat (1985) il est possible de dénombrer 6 principales formes de relance, réparties en deux catégories, les relances non directives, et les relances directives : les réitérations, les réitérations thématiques, les reformulations et les formulations du non-dit sont des relances non directives. Par contre, les questions spontanées ainsi que les questions préparées à l'avance insérées dans le guide d'entretien, sont des relances directives. Par ailleurs, selon Blanchet (1991) il existe deux registres dans la relance, le registre modal qui fonctionne comme un miroir, en reflet, il renvoie son discours à l'I $I^{e}$, en l'identifiant comme source. L'attitude de l' I' peut apparaître explicitement au travers d'expressions diverses comme « il me semble », «pour moi » ou rester implicite. Lorsque la relance est modale, l'attitude est reprise quand elle apparaît explicitement et révélée quand elle était implicite. Dans les deux cas la relance se fait en incluant des formules comme «selon vous », «à votre avis », "selon votre perception » etc. Le registre référentiel fonctionne en écho, il renvoie le contenu du discours. La reprise faite par l' $I^{\mathrm{er}}$ élide l'attitude de l' $\mathrm{I}^{\mathrm{e}}$ quand elle a été exprimée explicitement, et la laisse implicite si elle l'était au départ. La relance ne mentionne que les faits relatés. L'utilisation de chacun des ces registres à un effet sur le discours de $1^{\prime} \mathrm{I}^{\mathrm{e}}$. Le registre modal entraîne une modalisation du discours et se traduit par l'apparition de marques explicitant l'attitude (« il me semble» «pour moi »), parce qu'il est entendu par l' $I^{e^{\prime}}$ comme une remise en cause de sa sincérité (celui-ci est donc amené à réaffirmer que c'est bien là son point de vue). Cet effet peut être recherché lorsqu'il s'agit de mettre en évidence l'opinion des enquêtés. Le registre référentiel a pour conséquence une référentialisation du discours de l' $\mathrm{I}^{\mathrm{e}}$, parce que la relance est perçue comme une question indirecte visant un approfondissement. 
Pour toute citation : références à rappeler

Salès-Wuillemin, E. (2006). Méthodologie de l'enquête, in : M., Bromberg et A., Trognon (Eds.) Psychologie Sociale 1, Presses Universitaires de France, 45-77.

\subsubsection{Les relances propres à l'entretien non directif (END)}

L'END se caractérise par une grande souplesse donnée à l' ${ }^{\prime}{ }^{e}$ pour présenter ses contenus de pensée. Aucun cadre n'est préalablement établi et l' ${ }^{\mathrm{er}} \mathrm{s}$ 'ajuste totalement à son discours, il se contente de répéter ou de résumer ce que dit $1^{1} \mathrm{I}^{\mathrm{e}}$.

Les réitérations simples $(R S)$ sont les plus élémentaires, elles limitent les risques d'influence et sont aisées à produire parce qu'elles correspondent à une reprise de la dernière phrase ou partie de phrase au sein de la dernière prise de parole de l'I $I^{e}$. Elles sont interprétées comme des demandes indirectes d'explication (acte indirect de question, Searle, 1975) et contribuent à une linéarisation du discours en vertu de l'application des règles d'enchaînement (conditions thématique, de contenu propositionnel et illocutoire, Roulet, 1989). Les RS ne doivent toutefois pas être faites en trop grand nombre parce qu'elles donnent l'impression de n'être pas écouté. En effet, l'effort conversationnel réalisé par l' ${ }^{\mathrm{er}}$ semble être réduit au minimum, il s'agit d'une répétition sans travail d'organisation. Ayant le sentiment qu'il est seul à participer, l' ${ }^{\mathrm{e}}$ peut se sentir perdu et s'arrêter de parler. Nous présentons dans l'encadré suivant un exemple de RS avec déclinaison du registre modal et référentiel 17 .

Ié : oui, le manager doit être proche avec son équipe, sinon les gens osent pas lui demander...même si c'est son métier de t'aider...c'est le rôle de soutien métier, mais c'est aussi le travail du manager, le...soutien ...le soutien métier...alors il doit bien connaître le métier et être proche de l'équipe...pour aider quand il y a un besoin...

RS version modale : pour vous le manager doit avant tout aider quand il y a un besoin ... RS version référentielle : le manager doit aider quand il y a un besoin ...

Les réitérations thématiques $(R T)$ sont assez proches des RS. L'I ${ }^{\mathrm{er}}$ ne se contente toutefois pas de reprendre la dernière phrase de $1^{\prime} \mathrm{I}^{\mathrm{e}}$, il choisit une partie des propos de ce dernier, dans sa dernière prise de parole. Les RT donnent l'impression d'être moins mécaniques que les RS. L' $^{\text {er }}$ semble faire un effort conversationnel d'écoute plus important puisqu'il est capable de choisir et de répéter une phrase énoncée peu avant. Cependant, outre le risque d'influence résultant de la mise en exergue de certaines thématiques, le temps écoulé entre la formulation et la reprise entraîne des risques de déformation. Or, l'emploi d'un terme différent, même en apparence synonyme, peut induire une transformation du sens et une évolution de l'entretien : par

\footnotetext{
17 Les extraits des différents entretiens présentés portent sur la représentation du rôle du manager, regards croisés entre les managers et les managés : une étude pour France Télécoms (mémoire de DESS de psychologie sociale du travail, 2002, réalisé par Chritophe Monacci, sous la direction d'Edith Sales-Wuillemin).
} 
Pour toute citation : références à rappeler

Salès-Wuillemin, E. (2006). Méthodologie de l'enquête, in : M., Bromberg et A., Trognon (Eds.) Psychologie Sociale 1, Presses Universitaires de France, 45-77.

exemple, si l' I' dit «pour aider quand il y a un besoin » et que l' ${ }^{\mathrm{er}}$ reprend en disant «pour aider quand il y a des problèmes » il y a transformation du sens par réduction de la portée du référent.
$\mathrm{I}^{\mathrm{e}}$ : oui, il doit...aussi, oui ...il doit aussi savoir mener son équipe en étant juste et en respectant l'équipe et les problèmes des gens...oui il faut du respect, que le manager respecte chacun pour que ça les stimule...
RT version modale : votre sentiment c'est qu'il faut surtout $d u$ respect ...
RT version référentielle : Il faut du respect...

Les reformulations $(R E F)$ correspondent à un résumé de la dernière prise de parole de l' $\mathrm{I}^{\mathrm{e}}$. L' ' $\mathrm{I}^{\mathrm{er}}$ réalise un effort conversationnel plus important et donne l'impression qu'il intervient à part entière, qu'il «co-construit» (Ghiglione, 1986). Les REF présentent un important risque

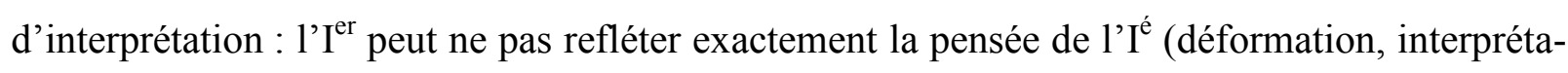
tion) ou ne pas respecter précisément la perspective en se focalisant sur des éléments qui n'avaient pas grande importance (amplification) ou en restreignant la portée d'autres éléments (réduction) ${ }^{18}$.
$\mathrm{I}^{\mathrm{e}}:$ Tous les problèmes que l'on peut rencontrer, avec le Service Informatique ou avec un client, le bon manager doit être là pour aider, et là il doit être proche, mais pas dans le même sens que tout à l'heure...proche...proche physiquement, n'être jamais très loin de son équipe de façon à être présent et disponible en cas de problème. Tu as vu, XX, il est jamais là, alors quand il y a un problème il faut lui courir après...et pendant ce temps le client c'est toi qu'il insulte...ça c'est chiant...
REF version Modale : Pour vous il est important que le manager soit très présent physiquement au sein de l'équipe pendant le travail...pour répondre immédiatement en cas de difficultés, sans $q u$ 'il soit besoin d'aller le chercher.
REF version Référentielle : Le manager doit donc être très présent physiquement au sein de l'équipe pendant le travail...pour répondre immédiatement en cas de difficultés, sans qu'il soit besoin d'aller le chercher.

Les Formulations du non-dit (FND) sont plus délicates à gérer. Ces relances s'appuient sur ce qui transparaît du discours sans en modifier la signification. Il s'agit d'expliciter l'implicite. Cela peut se faire en laissant transparaître l'attitude de l' I' (lorsque la FND est faite sur le registre modal) ou en tirant une conclusion à partir du contenu des propos de $1^{\prime} \mathrm{I}^{\mathrm{e}}$ (lorsque la FND est faite sur le registre référentiel). Parce que l'effort conversationnel de $1^{1} \mathrm{I}^{\mathrm{er}}$ semble maximal, les FND donnent une tournure extrêmement naturelle à la situation d'entretien. Les FND com-

\footnotetext{
18 Corrélativement, il y a le risque de faire un résumé trop précis, qui conduirait l' I'e à répondre par une validation totale : « oui, c'est ça, je suis d'accord avec vous » sans apporter d'autres éléments, ce qui peut gêner les interventions suivantes de $l^{\prime} I^{\text {er }}$.
} 
Pour toute citation : références à rappeler

Salès-Wuillemin, E. (2006). Méthodologie de l'enquête, in : M., Bromberg et A., Trognon (Eds.) Psychologie Sociale 1, Presses Universitaires de France, 45-77.

portent toutefois un risque énorme d'interprétation. En outre, elles peuvent être perçues par l' $\mathrm{I}^{\text {é }}$ comme une obligation d'assumer des conclusions ou des prises de position dont il voudrait peut être se garder, ce qui peut être ressenti comme une agression. Voilà pourquoi ces interventions nécessitent la plus grande prudence.

$\mathrm{I}^{\mathrm{e}}:$ Oui, proche de l'équipe, s'il est un membre de l'équipe, c'est mieux, et puis proche aussi juste être là, proche physiquement quand il y a des problèmes.

FND version Modale : Pour vous, un manager qui n'est pas membre de l'équipe ne peut être un bon manager

FND version Référentielle : L'équipe doit pouvoir faire appel à lui pour résoudre les problèmes...

\subsubsection{La conduite spécifique d'un Entretien Semi Directif (ESD)}

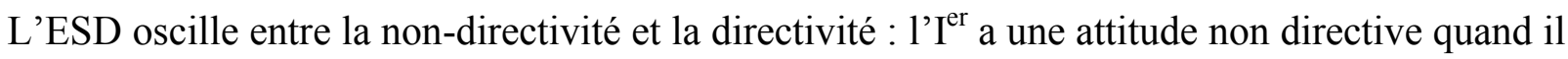
répète et reformule les propos de l'I $\mathrm{I}^{\mathrm{e}}$ grâce au RS, RT, REF, FND, et une attitude directive quand il pose des questions spontanées ou préparées. Nous ne présentons ici que les relances directives, les autres ayant déjà été détaillées.

Les questions spontanées (QS) sont totalement improvisées, précises et ajustées au discours de $1^{\prime} \mathrm{I}^{\mathrm{e}}$. Elles visent à éclaircir des éléments de contenu non explicités (questions référentielles) ou des aspects de l'attitude non précisés (questions modales).

$\mathrm{I}^{\mathrm{e}}$ : Ouais, ça devrait être un membre de l'équipe et pas quelqu'un qui est jamais là. T'as vu quand $X Z$ elle vient, tout le monde lui saute dessus, ben ça c'est parce que avant elle était tout le temps là. Et puis elle connaît tout, elle t'aidait et te soutenait... ben le soutien-métier quoi, tu vois...c'est quelqu'un qui t'aide et je crois que le manager devrait toujours aider les membres de son équipe. Et pas seulement relever les compteurs...Des fois j'ai l'impression que XX vient nous surveiller...

QS version référentielle ${ }^{19}$ : Qu'est-ce que vous voulez dire plus précisément par soutien-métier? QS version modale 20 : Comment vivez-vous cette situation?

Les questions préparées (QP) sont beaucoup plus générales. Elles sont préalablement formulées et insérées dans un guide d'entretien qui constitue un schéma directeur standardisé ${ }^{21}$ de l'entretien en limitant les risques de dispersion. Les QP s'utilisent dans des conditions strictes.

Une fois la consigne introductrice énoncée, l' I' $\mathrm{e}^{\mathrm{e}}$ prend la parole et s'exprime librement. Lorsque se produit une pause de plus de quelques secondes, $l^{\text {'er }}{ }^{\text {er }}$ relance ce premier propos «spontané » grâce à des interventions non directives et des QS. Ce n'est que lorsque cette partie d'entretien aura été suffisamment développée que la première QP pourra être posée. Une fois la question po-

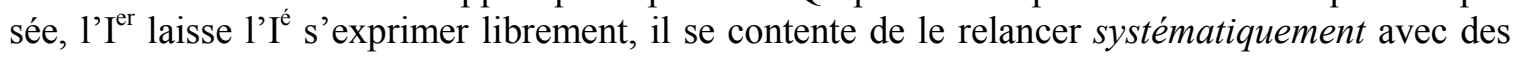

\footnotetext{
19 Question qui vise à une identification supplémentaire de la référence

${ }^{20}$ Question qui vise à une explicitation de l'attitude.

${ }^{21} \mathrm{~L}$ 'ordre et la formulation des questions ne varient pas d'un entretien à l'autre
} 
Pour toute citation : références à rappeler

Salès-Wuillemin, E. (2006). Méthodologie de l'enquête, in : M., Bromberg et A., Trognon (Eds.) Psychologie Sociale 1, Presses Universitaires de France, 45-77.

interventions non directives et des QS. Ce n'est que lorsque la thématique est suffisamment approfondie que la QP suivante peut être introduite. Si toutefois il apparaît que cette question a reçu un développement satisfaisant dans la partie précédente de l'entretien, il est possible de passer directement à la question suivante du guide, sans introduire celle qui était chronologiquement prévue.

L'élaboration du guide d'entretien est une étape importante de l'enquête : les erreurs sont à ce stade critiques parce qu'elles ont une incidence directe sur les données recueillies. Le guide se construit en 5 étapes.

1-évocation des thèmes qui structurent l'objet d'étude (étude de documents, " brain storming ", entretiens non directifs préalables ...) sont autant de sources possibles d'information ;

2-regroupement des thèmes dans un nombre limité de catégories ${ }^{22}$ (avec respect des règles de catégorisation : pertinence, exhaustivité, homogénéité, exclusivité) ;

3-intégration des thèmes généraux dans le guide ;

4-élaboration d'une ou de plusieurs questions par thème (selon le type d'entretien END ou ED). Celles-ci doivent rester de portée générale, leur formulation est proche d'une question inductrice ; 5-détermination de leur ordre d'introduction dans l'entretien. Plusieurs ordres possibles : logique (questions portant sur les causes, puis les conséquences, puis l'évolution), psychologique (questions les moins impliquantes en premier, les plus impliquantes en dernier), thématique (questions générales en premier plus spécifiques en dernier) etc.

Pour illustrer notre propos, nous présentons un guide d'ESD conçu pour répondre à une commande faite par une entreprise qui venait de recruter 200 jeunes diplômés afin d'opérer un rééquilibrage de la pyramide des âges. Ceci a cependant entraîné des difficultés d'intégration : selon la direction, les «nouveaux » témoignaient d'une moindre implication et une moindre adhérence aux projets de réforme de l'entreprise que les « anciens ». Les résultats de la pré-étude (entretiens non formalisés avec des jeunes/anciens collaborateurs et des managers) ont entraîné une reformulation de la question d'étude comme suit « les difficultés relevées peuvent-elles résulter d'une discrimination dont les nouveaux collaborateurs penseraient être la cible ? ». Deux sources de discrimination potentielle (VI théorique) pouvaient être relevées : leur inexpérience (ils sont nouveaux dans l'entreprise) leur âge (ils sont jeunes). La partition pouvait donc opposer les expérimentés/inexpérimentés ou les seniors/juniors. Dans cette perspective, l'hypothèse théorique que les collaborateurs juniors/inexpérimentés devaient être plus discriminés que les autres a été posée. 40 salariés répartis en 4 catégories ont été interviewés : juniors inexpérimentés, seniors inexpérimentés, juniors expérimentés, seniors et expérimentés ${ }^{23}$. Pour n'induire

\footnotetext{
224 à 6 en moyenne.

23 Opérationnalisation de la VI : ont été considérés comme juniors les individus ayant moins de 30 ans, les seniors avaient plus de 35 ans. Les collaborateurs inexpérimentés étaient en poste depuis moins d'un an, et les expérimentés depuis plus de 4 ans.
} 
Pour toute citation : références à rappeler

Salès-Wuillemin, E. (2006). Méthodologie de l'enquête, in : M., Bromberg et A., Trognon (Eds.) Psychologie Sociale 1, Presses Universitaires de France, 45-77.

aucun style discursif particulier la question inductrice des entretiens était formulée de la manière suivante : «J'aimerais que vous me parliez de vos collaborateurs, des collègues, des personnes qui travaillent à la $\mathrm{CEB}^{24} »$. Le guide d'entretien était structuré de la manière suivante.

Insérer ici Tableau 2

L'analyse des entretiens s'est faite par analyse thématique suivie d'une analyse de discours. Elle a permis de révéler que le positionnement «psychologique » des I ${ }^{\text {és }}$ n'était pas strictement équivalent à leur positionnement « objectif » (dû à l'âge et l'ancienneté). Par ailleurs, il est apparu que l'âge étaient une plus grande source de discrimination que l'inexpérience : conformément à l'hypothèse, les collaborateurs juniors inexpérimentés étaient plus discriminés que les seniors inexpérimentés, les juniors expérimentés plus que les seniors expérimentés.

\subsubsection{La conduite d'un entretien directif (ED) et l'élaboration du guide}

Dans un ED, l'enquêteur dirige totalement l'entretien. En théorie, il lui est possible de faire des relances non directives et directives, mais en pratique, il se contente le plus souvent de formuler les questions du guide, dans l'ordre préétabli. Sa forme Question/Réponse est donc très proche d'un questionnaire avec des questions ouvertes et il est difficile pour l' $\mathrm{I}^{\mathrm{e}}$ de sortir du cadre de référence défini à l'avance. Le guide d'entretien est construit de la même façon que pour l'ESD, simplement chacun des thèmes retenus génère plusieurs questions ${ }^{25}$.

\subsubsection{Les trois formes d'entretien et leurs utilisations : remarques méthodologiques}

Plus la marge de manoeuvre laissée à l'enquêté est faible et plus le chercheur doit avoir d'hypothèses précises. Autrement dit, l'END peut être utilisé dans une étude exploratoire sans qu'il y ait une connaissance approfondie du thème, de la population et une formulation d'hypothèses spécifiques. L'ESD et l'ED nécessitent à des degrés divers, une connaissance plus étendue de l'objet et de la population pour pouvoir construire un guide pertinent et des hypothèses spécifiques.

De manière générale il est possible de dire que l'entretien constitue une méthode de recueil très souple, qui peut être adapté à de multiples objectifs de recherche. Cette flexibilité ne doit toutefois pas faire oublier les limites de ce mode d'investigation. Plusieurs ensembles de biais peu-

\footnotetext{
24 étude réalisée pour la Caisse d'Epargne de Bourgogne, mémoire de Master Professionnel de psychologie du travail réalisé par Jenny Zanotti en 2002 sous la direction d'Edith Sales-Wuillemin.

25 En principe il y de 4 à 5 questions par thème, soit une moyenne de 15 à 20 questions par entretien.
} 
Pour toute citation : références à rappeler

Salès-Wuillemin, E. (2006). Méthodologie de l'enquête, in : M., Bromberg et A., Trognon (Eds.) Psychologie Sociale 1, Presses Universitaires de France, 45-77.

vent intervenir dans l'entretien, ils sont liés au lieu de recueil, aux caractéristiques de l' ${ }^{e r}$ et par suite à la distance sociale entre l' I' et l' l' I ainsi qu'à l'influence exercée par l' $I^{\mathrm{er}}$ sur l' $\mathrm{I}^{\mathrm{e}}$.

Comme le précisait Hymes (1962), l'espace dans lequel se déroule la communication a un effet sur ce qui se dit. Il recouvre le cadre physique (taille du local, bruits, odeurs, disposition des protagonistes ...) et psychologique, la perception que les protagonistes ont du cadre en raison de paramètres qui peuvent être visibles ou invisibles (comme la connaissance des activités qui s'y déroulent habituellement). Les caractéristiques de $1^{\text {'er }} \mathrm{I}^{\mathrm{er}}$ sont essentielles pour $1^{\text {' }} \mathrm{I}^{\mathrm{e}}$. Il a en effet besoin de calculer la distance sociale le séparant de l'Ier. Ce calcul prend en compte la classe sociale, les caractéristiques physiques, sexe, age etc. des protagonistes, plus ils diffèrent plus la distance est grande. Pour l'inférer, l' I' se fonde sur plusieurs indices (l'habillement, le langage employé, la gestuelle ...). Ce calcul permet à l' $I^{e}$ d'ajuster son discours au niveau du contenu et de la relation, par exemple, en le rendant accessible à l' ${ }^{\mathrm{e}}{ }^{\mathrm{er}}$, ou en calquant ses prises de position sur celles supposées de l' ${ }^{\mathrm{er}}$. Si la distance sociale lui apparaît trop importante, il peut conclure que $1^{\mathrm{I}} \mathrm{I}^{\mathrm{er}}$ ne sera pas capable de comprendre ce dont il parle, ou qu'il risque d'être réfractaire à ses propos; s'il la juge trop proche, il peut conclure que les informations ou les prises de position qu'il pourrait donner vont de soi, qu'il n'est pas besoin de les développer. En règle générale pour limiter ces biais sont préférées des situations où la distance sociale est moyenne. L'influence de l' $I^{\mathrm{er}}$ sur l' $\mathrm{I}^{\mathrm{e}}$ est grande en raison de l'adaptation de ce dernier, lorsque $1^{\prime} \mathrm{I}^{\mathrm{er}}$ fait des relances cet effet est amplifié comme l'ont montré expérimentalement Blanchet et al. 1991. Plus encore, d'après Matalon (1992) la situation elle-même de questionnement est par nature source d'influence, en effet le fait d'inviter les $\mathrm{I}^{\text {és }}$ à s'exprimer sur un objet peut les amener à construire une image (représentation, attitude ...) de cet objet. La question posée, la manière dont elle est posée, les incitent à y réfléchir alors qu'ils ne l'auraient peut être pas fait spontanément. Selon l'auteur, on est dès lors en droit de se demander si l'état intérieur de l'individu est toujours le même avant et après l'entretien et si les individus interrogés sont toujours représentatifs de la "masse silencieuse" qu'ils sont censés représenter ? Si tel n'est pas le cas, se pose le problème de la généralisation des résultats c'est-à-dire de la validité externe de la méthode.

Malgré ces multiples inconvénients la méthode d'entretien reste largement utilisée. Chacun s'accorde néanmoins à dire qu'elle ne peut être employée de manière isolée, un croisement systématique avec d'autres méthodes doit être opéré. 
Pour toute citation : références à rappeler

Salès-Wuillemin, E. (2006). Méthodologie de l'enquête, in : M., Bromberg et A., Trognon (Eds.) Psychologie Sociale 1, Presses Universitaires de France, 45-77.

\subsection{L'analyse des entretiens}

\subsubsection{Objectif et différentes phases de l'analyse}

L'analyse des données verbales vise à révéler le contenu des propos recueillis (mots prononcés, thématiques abordées ...), leur organisation (réseau thématique, organisation logique, ...), afin d'analyser les liens existant entre ces propos et l'état mental de l'individu (attitude, représentation ...). Il existe un grand nombre de techniques d'analyse, le traitement passe néanmoins toujours par 2 grandes phases : une phase de pré-analyse et une phase d'analyse. La pré-analyse comprend le choix et la transcription des documents analysés. L'analyse comporte une étape de découpage du texte (en unités) et une phase de catégorisation de ces unités (Cf. Bardin, 1991 ; Jakobi et al. 1994).

\subsubsection{Quelques exemples de méthode d'analyse}

Nous présentons trois méthodes : l'analyse lexicale, l'analyse thématique et l'analyse du discours.

L'analyse lexicale (cf. Lebart et Salem, 1988) consiste à repérer tous les mots et les expressions figées, présents dans les textes analysés, de les présenter par ordre décroissant de fréquence de citation dans des tableaux croisés, afin de mettre en évidence les différences existant entre les groupes d'individus. L'unité de découpage est le mot ou le groupe de $\operatorname{mots}^{26}$. Une analyse lexicale sans lemmatisation ${ }^{27}$ est réalisée par Sales-Wuillemin et al. (2005) concernant la représentation que les occupants d'un même immeuble d'Habitations à Loyer Modéré (HLM) ont de leur logement, en fonction de leur statut de locataires (12 occupants) ou de propriétaires (8 occupants) $)^{28}$.

\section{Insérer ici Tableau 3}

Ne sont retenus que les mots cités par plus de la moitié des $\mathrm{I}^{\text {ses }}$. La probabilité que 6/12 locataires ou que $4 / 8$ propriétaires produisent le même mot est de $\mathrm{p}<.00001$ (loi binomiale). Il s'agit de révéler les différences au niveau du lexique des deux populations. L'ampleur de ces différences peut être évalué grâce au test du $\chi^{2} \mathrm{ou}$, lorsque les effectifs sont restreints, comme c'est le cas ici, grâce au test exact de Fischer. Ces tests déterminent si la répartition des effec-

\footnotetext{
26 L'analyse peut être en partie automatisée grâce à l'utilisation de logiciels (cf. Marchand, 1998).

27 Regroupement des mots ayant un radical commun.

28 La technique utilisée ne repose sur aucune lemmatisation (à part les regroupements des formes au singulier et au pluriel).
} 
Pour toute citation : références à rappeler

Salès-Wuillemin, E. (2006). Méthodologie de l'enquête, in : M., Bromberg et A., Trognon (Eds.) Psychologie Sociale 1, Presses Universitaires de France, 45-77.

tifs est dépendante du hasard ou résulte de l'effet des VI. Par exemple, le terme « immeuble » est évoqué par $1 / 12$ des locataires et $5 / 8$ des propriétaires, le test exact de Fischer montre que cette différence est significative à $\mathrm{p}<.02$. De même le terme « respect » est évoqué par 4/8 des propriétaires et aucun locataire, le test exact de Ficher montre que cette différence est significative à $\mathrm{p}<.01$. Cependant, ce traitement ne permet pas de révéler les liens existant entre les mots produits par les $\mathrm{I}^{\mathrm{e}}$. Ceci peut se faire grâce à une analyse de similitude.

L'analyse de similitude permet de rendre compte de la proximité existant entre les mots produits par les $I^{\text {śs }}$ (éléments de la représentation). Elle peut être mesurée en demandant directement aux enquêtés d'effectuer une cotation, grâce à une échelle de distance, entre les mots présentés par paires (cf. infra). Cependant, cette technique étant artificielle, les auteurs ont reconstitué la matrice en évaluant la proximité établie spontanément par les I és au sein des entretiens $^{29}$. Deux représentations graphiques ont été réalisées à partir de la matrice des distances minimales («matrice des $3 »)$ : la classification hiérarchique et l'arbre maximum. Nous ne présentons ici que les résultats de l'arbre maximum pour le groupe des locataires. Le schéma représente un réseau, il se lit en partant du centre (grisé) puis en suivant les différentes ramifications. L'analyse présentée par les auteurs montre que la représentation des locataires s'organise autour de l'élément « voisins », celle des propriétaires autour de l'élément « immeuble ».

\section{Insérer ici Figure 2}

L'analyse thématique est la méthode la plus utilisée y compris dans l'enquête (cf. D’Unrug, 1974). Elle est dite ouverte parce qu'elle ne repose pas sur un cadre théorique préétabli, les résultats sont dus à la seule méthodologie (cf. Ghiglione et Matalon, 1991). Elle permet un premier contact exploratoire avec un objet en rendant compte de noyaux de sens ou thèmes qui ont une fréquence importante dans les discours. Le thème est un élément organisateur du discours, il correspond à une unité de sens implicite et sert à la fois d'unité de découpage et de catégorie. Le texte est réduit par le codeur à une suite d'expressions saillantes appelées Thèmes Secondaires (TS), chaque TS est affecté dans une catégorie plus vaste ou Thème Principal (TP). Le TP est désigné par un mot clef ou une expression renvoyant à un concept.

Les tableaux de résultats présentent les TP et éventuellement quelques exemples illustratifs de TS. Ils comportent 1-Des fréquences moyennes d'occurrence de chaque TP, c'est-à-dire le

\footnotetext{
${ }^{29}$ Il s'agissait de coter 0 les éléments de la paire lorsqu'ils n'apparaissaient pas dans le même entretien, 1 lorsqu'ils apparaissaient ensemble mais à des distances éloignée, 2 lorsqu'ils se manifestaient dans la même prise de parole, et 3 dans la même proposition grammaticale.
} 
Pour toute citation : références à rappeler

Salès-Wuillemin, E. (2006). Méthodologie de l'enquête, in : M., Bromberg et A., Trognon (Eds.) Psychologie Sociale 1, Presses Universitaires de France, 45-77.

nombre moyen de fois où le thème a été abordé dans les entretiens ; 2- la densité moyenne de chaque TP. Ce score est obtenu en divisant, pour chaque entretien, le nombre moyen de fois où le thème a été abordé par le nombre total de phrases (ou de propositions) de l'entretien. La densité moyenne donne un aperçu du «taux de recouvrement » des thèmes codés donc de la pertinence des catégories retenues ; 3- la proportion moyenne, correspond à la moyenne des scores obtenus en divisant le nombre de fois où le thème a été abordé dans chaque entretien par le nombre total d'occurrence de tous les thèmes abordés dans l'entretien. La proportion moyenne donne un aperçu de l'importance d'un thème par rapport à l'autre. Il est ainsi possible de les hiérarchiser.

L'analyse réalisée par Stewart et al. (2005) à propos de la représentation du logement en fonction du statut de l'occupant nous permet d'illustrer une utilisation de l'analyse thématique. Les scores (fréquences moyennes d'apparition des TP) sont comparés grâce au T de Student. L'analyse peut également inclure des valeurs positives ou négatives associées aux TP.

\section{Insérer ici Tableau 4}

La fréquence d'utilisation de l'analyse thématique ne doit toutefois pas faire oublier les problèmes qu'elle suscite. Ils apparaissent essentiellement lors de la constitution des catégories thématiques. Deux erreurs majeures sont à éviter. Tout d'abord l'augmentation exagérée du nombre de TP (qui résulte d'une tendance à rester trop près du texte, les TS et les TP se différencient insuffisamment), la solution est de restreindre le nombre de TP en augmentant leur niveau de généralité. La deuxième erreur est la réduction exagérée du nombre de TP (tendance à une interprétation exagérée donc à un éloignement du texte). La solution est d'augmenter le nombre de TP. Ces deux erreurs entraînent des problèmes de quantification : dans le premier cas les fréquences d'occurrence s'avèrent trop basses, dans le deuxième elles s'avèrent trop élevées. Dans les deux cas les différences se révèlent le plus souvent non significatives.

L'analyse de discours permet d'appréhender la relation existant entre l'I é et l'objet (cf. Ghiglione et al. 1985 ; Jacobi et al. 1994) grâce à des indicateurs comme les verbes, les déterminants, les conjonctions de coordination etc. L'avantage principal réside dans la non maîtrise de ces indicateurs par les $\mathrm{I}^{\mathrm{e} s}$. Elle autorise en outre un traitement par Anova. Dans l'étude de Sales-Wuillemin et al. (2005) la relation entre l'occupant et son logement transparaît dans la manière de le dénommer de façon directe (appartement, tanière, logement...) ou indirecte (ici, 
Pour toute citation : références à rappeler

Salès-Wuillemin, E. (2006). Méthodologie de l'enquête, in : M., Bromberg et A., Trognon (Eds.) Psychologie Sociale 1, Presses Universitaires de France, 45-77.

là ...), dans le choix des déterminants qui accompagnent ces mots (ma, mon, ce, ...). Un déterminant possessif et une désignation directe marquent une appropriation plus importante.

\section{Insérer ici Tableau 5}

L'analyse des résultats par ANOVA fait apparaitre que les propriétaires s'approprient plus leur logement que les locataires, ils utilisent un plus grand nombre de substantifs accompagnés d'un pronom possessif («mon» logement, «mon» appartement ...), cet effet est tendanciel $(F(1,18)=3.69 ; p<071)$. Les locataires se l'approprient moins, ils utilisent plus de désignations indirectes pour référer à leur logement, l'effet est significatif $(F(1,18)=5.07 ; p<.038)$.

\section{DEUXIEME PHASE DE L'ENQUETE : LE QUESTIONNAIRE}

Les questionnaires peuvent être auto administrés (remplis par l'enquêté) ou hétéro administrés (remplis par l'enquêteur sous la dictée de l'enquêté). La passation peut se faire de manière individuelle ou collective ; en face-à-face, par voie postale ou par Internet (cf. De Singly, 1992). Chacun de ces modes de passation comporte ses limites (par exemple, les passations collectives augmentent le nombre de réponses de conformisme, les passations par Internet restreignent les échantillons à une certaine catégorie de la population).

On dénombre 3 grandes catégories de questions : les questions d'identification, les questions cibles, les questions de remplissage. Les questions d'identification caractérisent l'enquêté, sexe, âge, profession, formation, etc. et positionnent les répondants en regard des VI et de leurs différentes modalités. Les questions cibles concernent directement l'objet de mesure, elles conduisent les enquêtés à positionner leur point de vue, à évoquer leurs connaissances etc. autant de réponses qui constitueront des indicateurs de la VD. Les questions de remplissage encadrent les questions cibles, elles harmonisent les transitions, élargissent l'objet d'étude, limitent les effets de halo, les biais de cohérence, les biais de réactance/complaisance, etc. Nous ne traitons dans cette partie que des questions cibles. Elles seront présentées en regard de la relation sujet/objet visée dans l'enquête (attitude, représentation ou préjugés/stéréotypes).

4.1 La mesure des attitudes : principaux modèles de question utilisables et modes de dépouillement des réponses 
Pour toute citation : références à rappeler

Salès-Wuillemin, E. (2006). Méthodologie de l'enquête, in : M., Bromberg et A., Trognon (Eds.) Psychologie Sociale 1, Presses Universitaires de France, 45-77.

Les questions se présentent sous la forme d'échelles, chacune permet de mesurer une caractéristique bien spécifique de l'attitude (cf. Alexandre, 1971).

\subsubsection{Echelle binaire adaptée de Thurstone (1929)}

Elle permet de mesurer la dimension cognitive de l'attitude, c'est-à-dire la définition de l'objet attitudinal. Sa construction se fait en 4 étapes : 1- Recension des assertions produites à propos de l'objet, au sein des entretiens, en ne conservant que les plus fréquentes ; 2- Regroupement en catégories de mêmes sens ; 3- Prototypisation des déclarations situées au sein d'une même catégorie, de façon à construire des formulations représentatives ; 4-Formulation des affirmations prototypiques comme modalités de réponses.

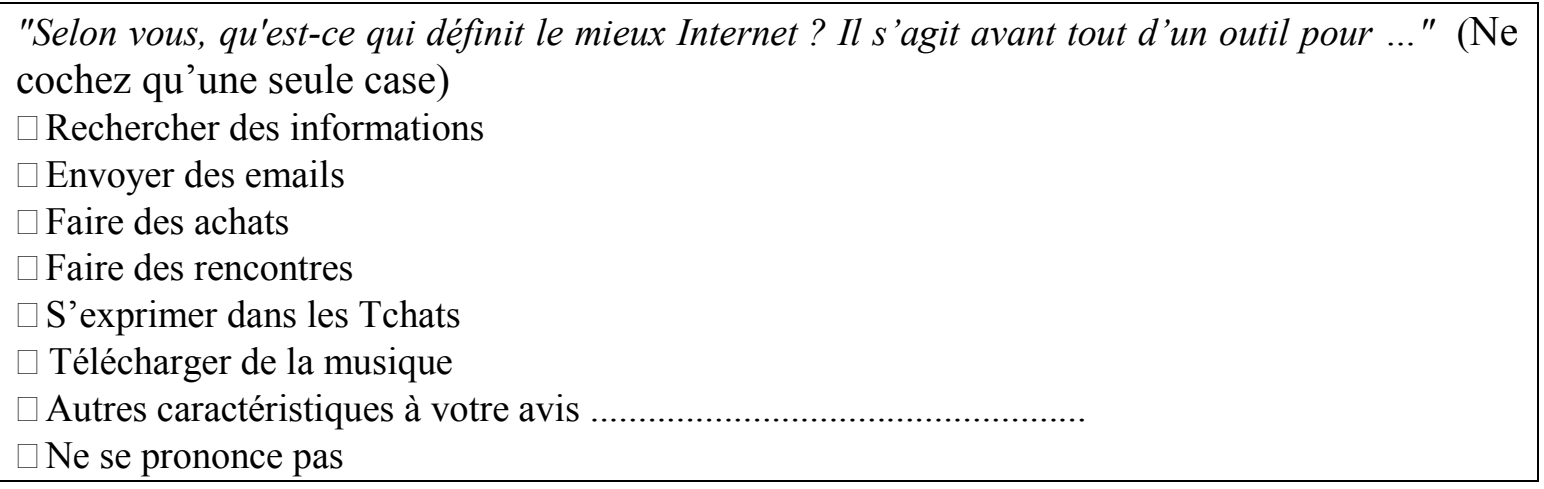

\subsubsection{Echelle bipolaire adaptée de Likert (1932)}

Elle mesure la dimension affective de l'attitude, à savoir les réactions positives ou négatives à l'égard de l'objet. Sa construction se fait en 3 temps 1- Recensement des prises de positions au sein des entretiens ; 2- Transformation éventuelle en questions ; 3- Association à un continuum bipolaire comportant un nombre impair d'échelons incluant un point 0 (attitude «neutre » ou «médiane ») et opposant deux points extrêmes acceptation/rejet, approbation/désapprobation etc.

«Quel est votre sentiment vis-à-vis de l'utilisation d'Internet dans les écoles primaires ?» (Entourez l'échelon qui correspond à votre position, sachant que si vous entourez le -2 cela veut dire que vous désapprouvez totalement et que si vous entourez le +2 vous approuvez totalement. Les échelons intermédiaires vous permettent de moduler votre jugement).

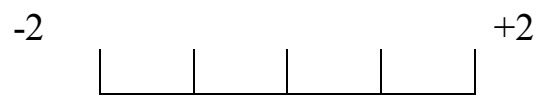

4.1.3 Echelle scalaire adaptée de Guttman (1950) 
Pour toute citation : références à rappeler

Salès-Wuillemin, E. (2006). Méthodologie de l'enquête, in : M., Bromberg et A., Trognon (Eds.) Psychologie Sociale 1, Presses Universitaires de France, 45-77.

Elle mesure la dimension conative de l'attitude. Elle a pour objectif de représenter les comportements de manière hiérarchique (scalogramme). L'image la plus proche est celle d'un thermomètre : l'adhésion à un degré supérieur implique celle à un degré inférieur. Par exemple, l'échelle du "militantisme politique" repose sur un engagement comportemental de plus en plus important.

«Répondez par oui ou par non en mettant une croix dans la case appropriée »

\begin{tabular}{|l|l|l|}
\hline & OUI & NON \\
\hline A- Avez-vous voté aux dernières élections présidentielles ? & & \\
\hline B- Avez-vous voté aux dernières élections municipales ? & & \\
\hline C- Vous considérez vous comme assez bien informés politiquement? & & \\
\hline D- Avez-vous des discussions politiques avec vos collègues? & & \\
\hline E- Avez-vous donné de l'argent à un parti? & & \\
\hline F- Avez-vous assisté à des réunions politiques ? & & \\
\hline G- Avez-vous essayé de convaincre des gens d'adhérer à un parti ? & & \\
\hline H- Avez-vous collé, avec une équipe, des affiches sans autorisation ? & & \\
\hline
\end{tabular}

4.2 La mesure des préjugés/stéréotypes/discrimination : principaux modèles de question utilisables et mode de dépouillement des réponses

Les préjugés traduisent la dimension évaluative d'une attitude à l'égard d'un groupe cible. Pour les mesurer il est possible d'utiliser des échelles bipolaires équivalentes à celle de Likert (1932) présentée ci-dessus pour marquer l'acceptation ou le rejet du groupe cible, nous n'y revenons pas. Nous présentons des échelles pour la mesure des stéréotypes et de la discrimination.

4.2.1 Mesure des stéréotypes grâce à une échelle composée d'adjectifs antithétiques

Ces échelles utilisent le différenciateur stéréotypique adapté d'Osgood et al. 1957. Elles sont standardisées et s'appliquent à tous les groupes. Pour éviter les biais repérés classiquement dans la formulation des réponses (effet de primauté, de récence, effet de halo ...), l'ordre d'apparition des adjectifs et la valeur positive ou négative sont alternés par randomisation ou contrebalancement. Pour une adaptation à un groupe spécifique, il est possible de les construire en 3 étapes : 1- Réalisation d'entretiens ou de questions d'évocation ; 2- Identification des adjectifs évoqués ; 3- Insertion dans le questionnaire et appariement avec leurs opposés.

«Comment positionnerez-vous les membres de la communauté XXX sur les échelles suivantes. (Répondez en mettant une croix sur l'une des 5 cases proposées). 
Pour toute citation : références à rappeler

Salès-Wuillemin, E. (2006). Méthodologie de l'enquête, in : M., Bromberg et A., Trognon (Eds.) Psychologie Sociale 1, Presses Universitaires de France, 45-77.

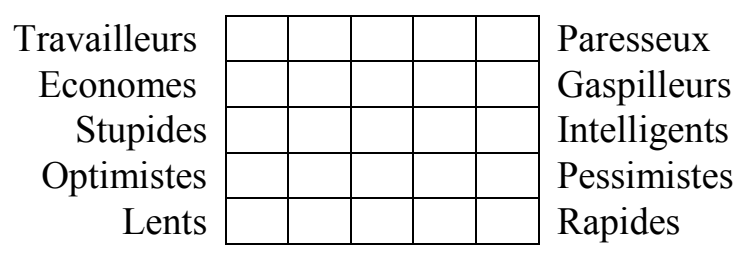

4.2.2 Mesure de la discrimination grâce à une échelle ordinale reposant sur un indicateur de mise à distance croissante : adaptée de Bogardus (1928) 30

«Laquelle de ces affirmations reprendriez-vous à votre compte? » (ne cochez qu'une seule case)

1 Je trouverais normal qu'un membre de la communauté ....... devienne mon proche parent par alliance et entre, par mariage, dans ma famille (qu'il devienne mon gendre ou mon beau-frère par exemple).

2 J'accepte d'avoir un membre de la communauté ......... pour ami personnel mais je n'aimerais pas qu'il fasse partie, par alliance, de ma famille.

3 Je n'attacherais aucune importance à ce qu'un membre de la communauté ......... devienne mon voisin en emménageant dans un appartement de l'immeuble ou j'habite, mais je ne chercherais pas à établir avec lui des relations de voisinage.

4 Je trouverais naturel de faire occasionnellement des achats dans un magasin dont le patron ou un employé sont membres de la communauté

5 J'admets que les membres de la communauté des citoyens à part entière.

6 Je trouverais normal que les membres de la communauté .......... aient un statut particulier dans mon pays et qu'ils ne soient pas considérés comme des citoyens à part entière.

7 A mon avis, les membres de la communauté ......... devraient être traités comme des ressortissants étrangers sur notre territoire et être soumis aux mêmes obligations que les autres étrangers.

8 Je suis d'accord pour que les membres de la communauté soient interdits de séjour sur le territoire national et que ceux qui y demeurent soient expulsés.

9 Je pense qu'il faut exterminer les membres de la communauté ici et ailleurs.

4.3 La mesure des représentations : principaux modèles de question utilisables et modes de dépouillement des réponses

Plusieurs techniques permettent de mesurer les représentations sociales. Les ouvrages de Doise et al. (1992), Moliner et al. (2002), Abric (2003) en donnent un aperçu très précis.

\footnotetext{
30 Dans cette étude, 1725 américains Blancs sont interrogés à propos de 40 groupes communautaires (anglais, français, suisses, norvégiens ...).
} 
Pour toute citation : références à rappeler

Salès-Wuillemin, E. (2006). Méthodologie de l'enquête, in : M., Bromberg et A., Trognon (Eds.) Psychologie Sociale 1, Presses Universitaires de France, 45-77.

\subsubsection{Les questions d'associations verbales}

Dans l'association verbale simple, il s'agit de donner les premiers mots qui viennent à l'esprit, à partir de l'inducteur présenté (mot qui désigne l'objet de la représentation).

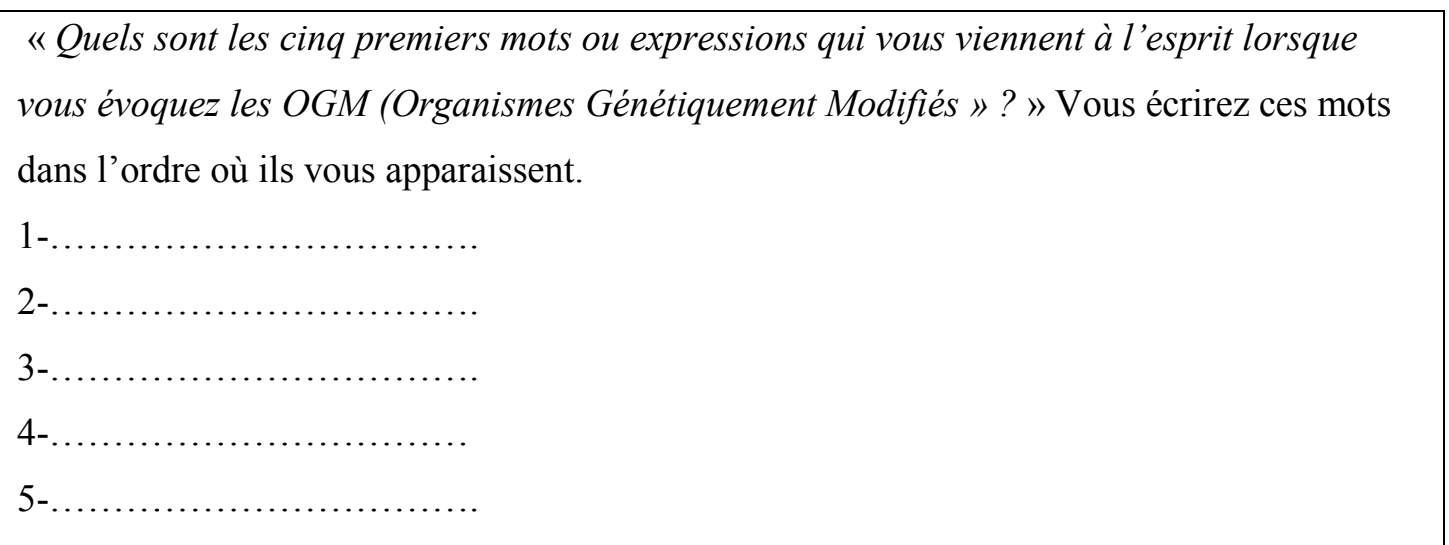

Le traitement suppose une analyse lexicale. L'analyse statistique peut se faire par $\chi 2$, test exact de Fischer, analyse de similitude (classification hiérarchique ou arbre maximum) que nous avons abordés précédemment, ou par Analyse Factorielle de Correspondance (AFC). Pour augmenter la lisibilité du graphique, seuls les mots les plus précocement et massivement évoqués par les enquêtés sont inclus dans l'analyse.

L'association verbale en dyades et triades vise une approche plus précise du sens à donner au mot induit, il s'agit de révéler des chaînes associatives à partir de couples ou de triades de mots inducteurs. Cette technique est utilisée par Guimelli (1994) sur la fonction d'infirmière. Elle se déroule en 3 phases : 1/Mise en évidence d'une chaîne associative à partir d'un mot inducteur; 2/ Association systématique du mot inducteur avec chacun des mots induits de la chaîne associative pour constituer une nouvelle chaîne associative ; 3/ Même procédé, mais cette fois-ci à trois éléments, c'est-à-dire avec des triades de mots inducteurs. La représentation des résultats se fait sous la forme d'un schéma que l'on appelle « carte associative » qui permet de visualiser les relations entre les éléments (cf. Abric, 1994).

\subsubsection{Les choix successifs par blocs}

Cette méthode est utilisée par Guimelli dans l'étude présentée en 1989 à propos de l'effet des pratiques nouvelles (de gestion et de préservation du territoire) sur la représentation de la chasse, chez un groupe de chasseur. Sont tout d'abord réalisés des ESD, puis les items les plus 
Pour toute citation : références à rappeler

Salès-Wuillemin, E. (2006). Méthodologie de l'enquête, in : M., Bromberg et A., Trognon (Eds.) Psychologie Sociale 1, Presses Universitaires de France, 45-77.

fréquemment cités sont insérés dans un questionnaire. 128 chasseurs répartis en 3 groupes sont interrogés : sujets n'ayant jamais mis en œuvre ces pratiques nouvelles, sujets très impliqués dans ces pratiques, et sujets mettant en œuvre ces pratiques de façon intermittente. Les enquêtés doivent repérer, parmi les 20 proposés, les 4 items qui correspondent le mieux à leur conception de la chasse et leur affecter le score +2 . Les 4 items qui à leur sens sont le plus éloignés reçoivent le score -2 . Au milieu, les 4 items relativement proches de leur conception, sont notés +1 et ceux qui sont relativement éloignés sont notés -1 . Enfin ceux qui ne sont ni acceptables ni rejetables sont notés 0 . Chaque item a ainsi un score sur une échelle de valeurs à 5 modalités ($2,-1,0,+1,+2)$. Les items à coter vont d'une conception traditionnelle de la chasse (« chasser c'est revenir chez soi avec du gibier », «être adroit et bien tirer », « utiliser légalement une arme », etc.) à une conception plus moderne («chasser c'est un moyen de connaître les habitudes du gibier », « gérer un territoire », « financer la protection de la nature », « respecter les animaux » etc.). Le traitement des résultats se fait par analyse de similitude. En appliquant cette méthode, l'auteur montre que ce sont les chasseurs ayant participé aux pratiques nouvelles qui considèrent comme positifs les items liés à la gestion du territoire. L'ensemble de ces items est au contraire considéré comme négatif par les chasseurs n'ayant jamais participé aux activités nouvelles, ces éléments sont néanmoins présents dans leur représentation.

\subsubsection{Les matrices d'évaluation pairées}

Cette technique a été utilisée par Le Bouedec (1984), elle est censée révéler la trame primitive (ou trame fondamentale) de la représentation. Ces matrices présentent les items les plus cités sous forme de couples obtenus au moyen d'un croisement de chaque item avec chacun des autres. Les enquêtés sont invités à coter la distance existant entre les termes de la paire à l'aide d'une échelle en 4 points : 0 si les deux mots ne vont pas ensemble, puis de 1 à 3 selon la force du lien, s'il existe. La cotation à 3 renvoyant au lien le plus fort. Le traitement des réponses se fait par analyse de similitude.

\subsection{Pré-test et passation du questionnaire}

Une fois les questions cibles formulées, seront insérées les questions de remplissage qui assureront la transition. Il est également utile de prévoir de rassembler les questions dans différentes parties qui pourront être nommées. Ces indications constituent une sorte de balisage et favorisent la progression dans le questionnaire. 
Pour toute citation : références à rappeler

Salès-Wuillemin, E. (2006). Méthodologie de l'enquête, in : M., Bromberg et A., Trognon (Eds.) Psychologie Sociale 1, Presses Universitaires de France, 45-77.

Une fois le questionnaire finalisé, il devra être pré-testé auprès de quelques enquêtés appartenant à la population visée. Lors de la passation ils seront invités à exprimer à voix haute leurs réactions ou interrogations. Un enregistrement est conseillé. Lors de l'analyse de ces données, une attention toute particulière devra être portée à la facilité des la progression dans le questionnaire, à la cohérence et la formulation des questions. Une fois les corrections réalisées, la passation finale pourra avoir lieu.

5. la communication des résultats : le rapport d'enquête

Le rapport d'enquête doit permettre de communiquer les résultats à des tiers. Parfois c'est cette phase qui se révèle la plus importante car elle donne toute la mesure du travail effectué, souvent long et difficile. Un rapport d'enquête mal présenté peut faire perdre beaucoup de crédit à l'équipe de travail. Il se compose tout d'abord d'une introduction générale qui spécifie les objectifs de l'étude, la question d'étude et son intérêt (théorique ou empirique). Ensuite est présenté le cadre théorique dans lequel le concept auquel est rattaché l'objet d'étude est intégré. A la fin de cette partie sont présentées la problématique et les hypothèses. La partie suivante correspond à la description de la méthode de recueil, c'est-à-dire les variables, la population, les outils (entretiens/questionnaires) et toutes les informations concernant la conception, le pré-test et la passation. Ensuite sont présentés la méthode d'analyse et les résultats de l'étude. Ces derniers sont associés à un commentaire et une interprétation. Enfin, la conclusion reprend les principaux résultats et propose une ouverture. En principe des propositions concernent une suite à donner au travail réalisé, voire une concrétisation des résultats en termes de préconisations.

\section{Conclusion}

La méthode d'enquête est un outil souple de recueil de données qui vise une mise au jour des contenus de pensée mais également une analyse de l'activité mentale. Lorsqu'elle est utilisée de manière raisonnée, elle s'avère très pertinente. Elle doit toutefois être complétée grâce à d'autres méthodes comme l'observation et l'expérimentation.

\section{Lectures conseillées}

Abric, J.C. (2003) (Ed.) Méthode d'étude des représentations sociales, Ramonville : Erès. Blanchet, A. (1991). Dire et faire dire : l'entretien, Paris : Colin (deuxième édition 1997). 
Pour toute citation : références à rappeler

Salès-Wuillemin, E. (2006). Méthodologie de l'enquête, in : M., Bromberg et A., Trognon (Eds.) Psychologie Sociale 1, Presses Universitaires de France, 45-77.

Chauchat, H., (1985), L'enquête en psychosociologie, Paris : Presses Universitaires de France.

Ghiglione, R., Matalon, B., (1978), Les enquêtes sociologiques, Paris : A. Colin (deuxième et troisième édition, 1980, 1991). 
Pour toute citation : références à rappeler

Salès-Wuillemin, E. (2006). Méthodologie de l'enquête, in : M., Bromberg et A., Trognon (Eds.) Psychologie Sociale 1, Presses Universitaires de France, 45-77.

TABLEAUX ET FIGURES A INSERER DANS LE TEXTE

Figure 1 : DEMARCHE GENERALE POUR LA REALISATION D'UNE ENQUETE PAR ENTRETIEN ET QUESTIONNAIRE

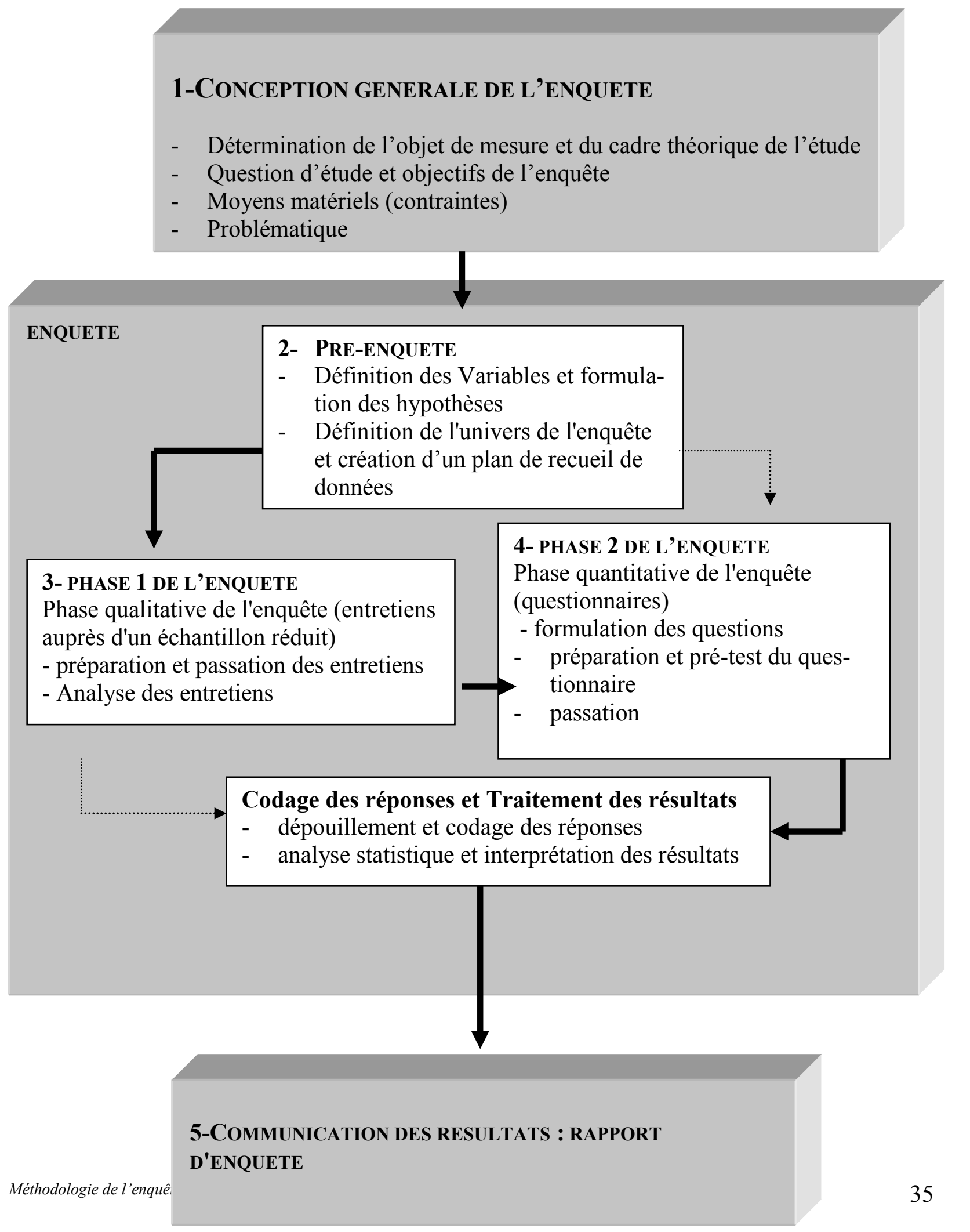


Pour toute citation : références à rappeler

Salès-Wuillemin, E. (2006). Méthodologie de l'enquête, in : M., Bromberg et A., Trognon (Eds.) Psychologie Sociale 1, Presses Universitaires de France, 45-77.

Tableau 1 : Plan de recueil de données à 2 VI : la représentation des malades atteints du Sida en fonction du « contexte d'acquisition des connaissances » et de la « valorisation de la cible ».

\begin{tabular}{|l|l|l|}
\hline Population interrogée/ Cible & Elèves infirmières & Elèves Assistantes Sociales \\
\hline Toxicomanes, prostitués & 50 & 50 \\
\hline Hémophiles, transfusés & 50 & 50 \\
\hline
\end{tabular}

$\underline{\text { Tableau } 2}$ : Exemple de guide d'ESD (sont mis en italique les parties de questions sur lesquelles l'Interviewer faisait porter un focus intonatif).

\begin{tabular}{|l|l|}
\hline Thèmes à aborder par les sujets & Questions correspondantes \\
\hline LES NOUVEAUX & $\begin{array}{l}\text { Pourriez-vous me parler des collaborateurs, des collègues qui } \\
\text { travaillent depuis peu à la CEB ? Quelle image en avez-vous ? }\end{array}$ \\
\hline LES ANCIENS & $\begin{array}{l}\text { Pourriez-vous me parler des collaborateurs, des collègues qui } \\
\text { travaillent depuis longtemps à la } C E B \text { ? Quelle image en avez- } \\
\text { vous? }\end{array}$ \\
\hline LES PLUS JEUNES & $\begin{array}{l}\text { Pourriez-vous me parler des collaborateurs, des collègues les } \\
\text { plus jeunes de la CEB } \text { ? Quelle image en avez-vous ? }\end{array}$ \\
\hline LES PLUS AGES & $\begin{array}{l}\text { Pourriez-vous me parler des collaborateurs, des collègues les } \\
\text { plus âgés de la } C E B \text { ? Quelle image en avez-vous ? }\end{array}$ \\
\hline
\end{tabular}

Tableau 3 : Mots et expressions les plus cités par les deux groupes d'occupants : locataires versus propriétaires. Les effectifs sont présentés en fréquence brute ${ }^{31}$ et en pourcentages (d'après Sales-Wuillemin et al. 2005).

\begin{tabular}{|l|l|l|l|l|r|r|}
$\mathbf{1 2}$ locataires & Fc de citation & $\mathbf{9}$ & & $\mathbf{8}$ propriétaires & Fc de citation & \% \\
\hline voisins & 10 & 0,83333333 & 1 & propriétaire & 7 & 0,875 \\
\hline Problème(s) & 9 & 0,75 & 2 & appartement & 6 & 0,75 \\
\hline travaux & 8 & 0,66666667 & 3 & problème(s) & 6 & 0,75 \\
\hline gens & 7 & 0,58333333 & 4 & acheté & 5 & 0,625 \\
\hline enfants & 7 & 0,58333333 & 5 & gens & 5 & 0,625 \\
\hline baignoire & 6 & 0,5 & 6 & logement & 5 & 0,625 \\
\hline Chenôve & 6 & 0,5 & 7 & l'immeuble & 5 & 0,625 \\
\hline cher & 6 & 0,5 & 8 & Personne(s) & 5 & 0,625 \\
\hline jeunes & 6 & 0,5 & 9 & voisinage & 5 & 0,625 \\
\hline logement & 6 & 0,5 & 10 & jeunes & 5 & 0,625 \\
\hline salle-de-bain & 6 & 0,5 & 11 & Chenôve & 4 & 0,5 \\
\hline appartement & 6 & 0,5 & 12 & locataires & 4 & 0,5 \\
\hline & & & 13 & mettre & 4 & 0,5 \\
\hline & & & 14 & propre & 4 & 0,5 \\
\hline & & & 15 & respect & 4 & 0,5 \\
\hline
\end{tabular}

31 Il s'agit du nombre de sujets ayant prononcé les mots et non du nombre d'occurrence moyen de chaque mot. 
Pour toute citation : références à rappeler

Salès-Wuillemin, E. (2006). Méthodologie de l'enquête, in : M., Bromberg et A., Trognon (Eds.) Psychologie Sociale 1, Presses Universitaires de France, 45-77.

\begin{tabular}{|l|l|l|l|r|r|}
\cline { 3 - 5 } & & 16 & ville & 4 & 0,5 \\
\hline & & 17 & voisins & 4 & 0,5 \\
\hline
\end{tabular}

$\underline{\text { Figure 2 }}$ : Organisation de la représentation des Locataires (d'après Sales-Wuillemin et al. 2005)

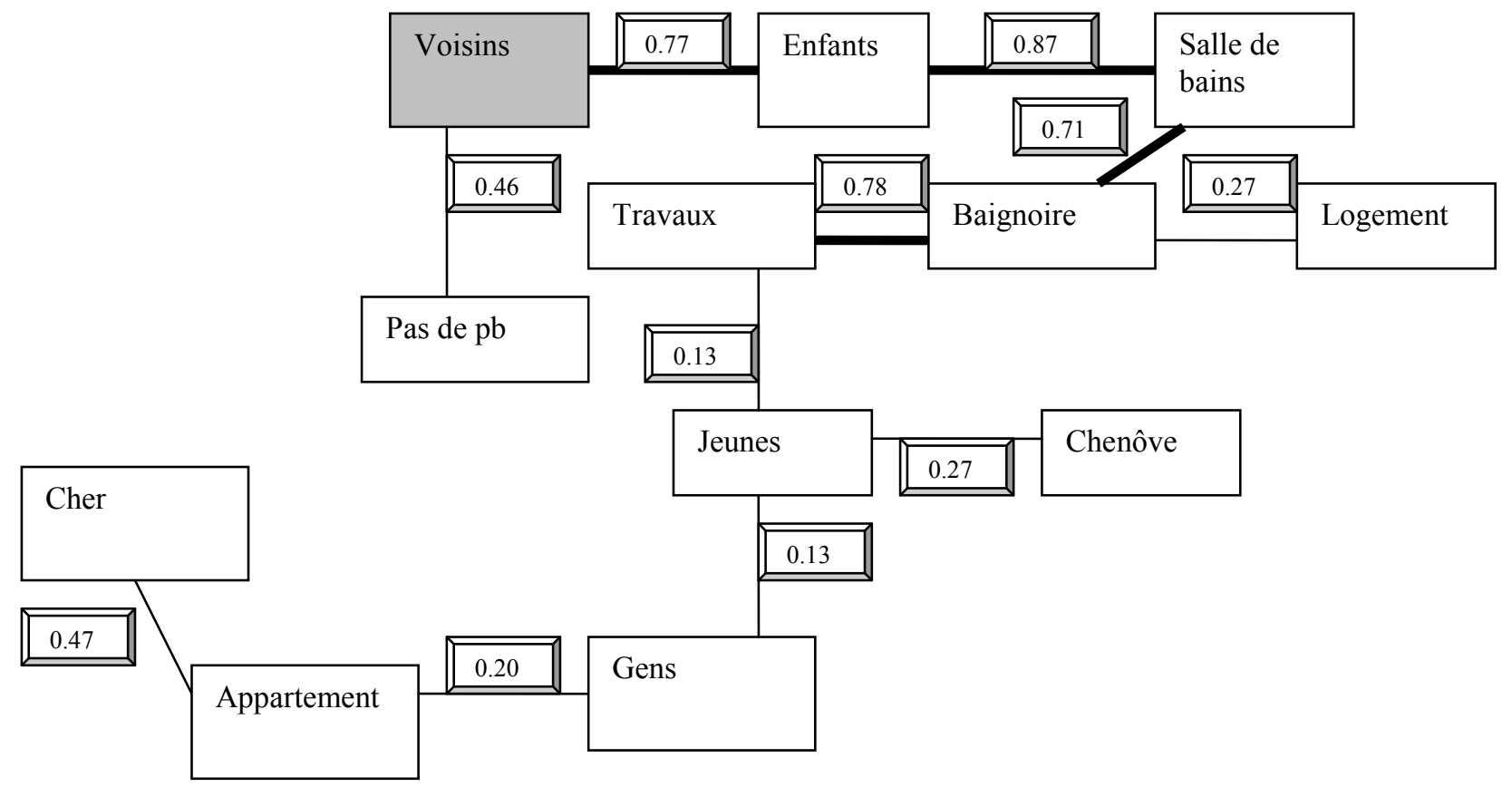

Tableau 4 Termes positifs (bien, plaire, aimer, gentil, sympa ...) et négatifs ((cher/trop élevé, sale, pas de respect, pas bien...) dans le discours des locataires et des propriétaires selon les différents TP (nombre moyen d'occurrences, en densité). En gras figurent les différences significatives $(\mathrm{p}<.05)$ au $\mathrm{t}$ de Student $(\mathrm{ddl}=22)$. D'après Stewart et al. (2005)

\begin{tabular}{|l|c|c|cc|}
\hline $\begin{array}{c}\text { Termes positifs at- } \\
\text { tachés aux TP }\end{array}$ & Locataires & Propriétaires & $\mathbf{t}^{*}$ & $\mathbf{p}$ \\
\hline Le logement & 6,77 & 6,45 & 0,513 & $\mathrm{~ns}$ \\
L'immeuble & 0,08 & $\mathbf{1 , 5 5}$ & $-2,421$ & $<.05$ \\
L'environnement & 0,23 & 0,82 & $-1,857$ & $<.10$ \\
Les voisins & 2,62 & 3,45 & $-0,408$ & $\mathrm{~ns}$ \\
Rel. société gérante & 0,46 & - & - & - \\
Travaux & 0,38 & - & - & - \\
Autres thèmes & 0,46 & 0,54 & $-0,641$ & $\mathrm{~ns}$ \\
\hline total & 11,00 & 12,81 & & \\
\hline Termes négatifs at- & Locataires & Propriétaires & $\mathbf{t} *$ & $\mathbf{p}$ \\
\hline \multicolumn{1}{c|}{ tachés aux TP } & 1,92 & 0,91 & 1,017 & $\mathrm{~ns}$ \\
\hline
\end{tabular}


Pour toute citation : références à rappeler

Salès-Wuillemin, E. (2006). Méthodologie de l'enquête, in : M., Bromberg et A., Trognon (Eds.) Psychologie Sociale 1, Presses Universitaires de France, 45-77.

\begin{tabular}{|l|c|c|cc|}
\hline L'immeuble & 0,69 & 1,27 & $-0,223$ & ns \\
\hline Les voisins & 0,31 & $\mathbf{2 , 3 6}$ & $-2,053$ & $<.06$ \\
\hline Travaux & 0,54 & - & - & - \\
\hline Autres thèmes & 0,61 & 0,45 & 0,557 & ns \\
\hline total & 4,07 & 4,99 & & \\
\hline
\end{tabular}

$\underline{\text { Tableau } 5}$ : Référence au logement a/ utilisation du pronom personnel « mon » pour accompagner le terme référant au logement personnel (logement, appartement, tanière ...); $b$ / d'une désignation indirecte (ici, là, ...). D'après Sales-Wuillemin et al. (2005)

\begin{tabular}{|l|l|l|}
\hline Statut & $\begin{array}{l}\text { Substantif accompagné d'un pro- } \\
\text { nom possessif (mon, ma ...) }\end{array}$ & $\begin{array}{l}\text { Désignation indirecte } \\
\text { (ici, là) }\end{array}$ \\
\hline Locataires & 0,5 & $4,25^{*}$ \\
\hline Propriétaires & $1,75^{*}$ & 2 \\
\hline
\end{tabular}

\section{BIBLIOGRAPHIE}

Abric, J.C. (2003) (Ed.) Méthode d'étude des représentations sociales, Ramonville : Erès.

Abric, J.C., (1989), L'étude expérimentale des représentations sociales, in : Jodelet, D. (Ed.), Les représentations sociales, Paris : Presses Universitaires de France, 189-203.

Alexandre, V. (1971). Les échelles d'attitudes, Paris : Editions Universitaires.

Allport, G.W., (1935), Attitudes, in C. Murchison, Handbook of social psychology, Worcester, Mass : Clark University Press.

Argentin, G., Ghiglione, R. (1986). Le système de communication, in : R. Ghiglione (1986) (Ed.) L'homme communiquant, Paris : A. Colin, 171-195

Bardin, L., (1979), L'analyse de contenu, Paris, Presses Universitaires de France, (2ème édition, 1991)

Blanchet, A. (1991). Dire et faire dire : l'entretien, Paris : Colin (deuxième édition 1997).

Blanchet, A. (Ed.), (1985), L'entretien dans les sciences sociales, Paris, Dunod.

Blanchet, A. Gotman, A., (1992), L'enquête et ses méthodes : l'entretien, Paris : Nathan Université, collection sociologie 128.

Blanchet, A., Bromberg, M., Urdapilleta, I. (1991). L'influence non directive, Psychologie Française, 35-3, 217-226.

Blanchet, A., Ghiglione, R., Massonat, J., Trognon, A., (1987), Les techniques d'enquêtes en sciences sociales, Paris, Dunod.

Bogardus, E.S. (1925). Measuring social distance. Journal of Applied sociology, 9, 299-308. 
Pour toute citation : références à rappeler

Salès-Wuillemin, E. (2006). Méthodologie de l'enquête, in : M., Bromberg et A., Trognon (Eds.) Psychologie Sociale 1, Presses Universitaires de France, 45-77.

Bourhis, R.Y., Leyens, J.P. (Eds.) (1999). Stéréotypes, discrimination et relations intergroupes, Liège : Mardaga.

Bouvron, A.M., Argentin, G., Jakobi, J.M (1986). Subordinations et insubordinations, in : R. Ghiglione (1986) (Ed.) L'homme communiquant, Paris : A. Colin, 113-169.

Bromberg, M. (1990). La communication : Le "Pourquoi". In : R. Ghiglione, C. Bonnet, J.F. Richard, (Eds.). Traité de Psychologie Cognitive, Tome 3, Cognition, représentation, communication, Paris : Dunod, 227-274.

Bromberg, M., Dubois, M. (1996). L'étude de la persuasion. In : J.C. Deschamps, J.L. Beauvois (Eds.). Des attitudes aux attributions : sur la construction de la réalité sociale. Grenoble : Presses Universitaires de Grenoble, 67-90.

Chauchat, H., (1985), L'enquête en psychosociologie, Paris : Presses Universitaires de France.

D’Unrug, M.C., (1974), L'analyse de contenu, Paris : Encyclopédie universitaire, Ed. Universitaires.

Doise, W., Clémence, A., Lorenzi-Cioldi, F. (1992). Représentations sociales et analyse de données. Grenoble : Presses Universitaires de Grenoble.

Dautun, M., Sales-Wuillemin, E., Morlot, R., Talon, D., Minariy-Dohen, P. (2005). Effets de la tâche et de la distance à l'objet, sur la mobilisation d'une représentation sociale : l'exemple de l'hygiène à l'hôpital. Contribution à la lutte contre les infections nosocomiales, Actes du 7ème Colloque International de Psychologie Sociale Appliquée, ADRIPS Rennes, France, 20- 22 Octobre 2005, 20p.

De Singly, F., (1992), L'enquête et ses méthodes : le questionnaire,Paris : Nathan université, collection sociologie 128 .

Delhomme, P., Meyer, T. (1997). Les projets de recherche en psychologie sociale, Paris : Colin, Collection cursus.

Eagly, A.H., Chaicken, S. (1998). Attitude Structure and Functions, in: D. Gilbert, S. Fiske, G. Lindzey (Eds.), The handbook of Social Psychology (4th ed., vol. 1). New-York: McGraw-Hill, 269-322.

Festinger, L., (1957), A theory of cognitive dissonance. Standford, CA : Stanford University Press.

Flament, C., (1989). Structure et dynamique des représentations sociales, in : Jodelet, D., (Ed.), Les représentations sociales, Paris, Presses Universitaires de France, 204-219. 
Pour toute citation : références à rappeler

Salès-Wuillemin, E. (2006). Méthodologie de l'enquête, in : M., Bromberg et A., Trognon (Eds.) Psychologie Sociale 1, Presses Universitaires de France, 45-77.

Ghiglione, R. (1986). La communication morcelée, in : R. Ghiglione (1986) (Ed.) L'homme communiquant, Paris : A. Colin, 9-33.

Ghiglione, R. (1988). Enjeux et discours : le paradigme de la cohérence, Revue Internationale de Psychologie Sociale, 1, 3-4, 365-388.

Ghiglione, R., Bromberg, M., Dorna, A. (1986). Parole et persuasion : les enjeux sociaux, in : R. Ghiglione (Ed.), L'homme communiquant, Paris : A. Colin, 77-109.

Ghiglione, R., Matalon, B., (1978), Les enquêtes sociologiques, Paris : A. Colin (deuxième et troisième édition, 1980, 1991).

Ghiglione, R., Matalon, B., Bacri, N., (1985), Les dires analysés : l'Analyse Propositionnelle $d u$ Discours, Paris : Presses Universitaires de Vincennes.

Goffman, E. (1959). The presentation of self in everyday life. Doubleday, New York. Traduction Française, (1973, 2ème edition 1987). La mise en scène de la vie quotidienne : 1-La présentation de soi, Paris : Editions de Minuit.

Gosling, P. (Eds.) (1996). Psychologie sociale, Paris : Bréal.

Grice, H.P., (1975), Logic and conversation, in: P. Cole (Ed.), Syntax and Semantics, vol. 3, Speech Acts, New-York : Academic Press, 41-58. (trad. fr. (1979), Logique et conversation, Communications, 30, 57-72.

Guimelli, C., (1989), pratiques nouvelles et transformation sans rupture d'une représentation sociale : la représentation de la chasse et de la nature, in : Beauvois, Joulé, Monteil, (Eds.), Perspectives cognitives et conduites sociales, Tome 2, Cousset, Delval, 117-138.

Guimelli, C., et Rouquette, M.L., (1992). Contribution du modèle associatif de Schèmes Cognitifs de Base à l'analyse structurale des représentations sociales. Bulletin de Psychologie. XLV, 405, 196-202.

Guimelli, Ch. (1994). Transformation des représentations sociales, pratiques nouvelles et schèmes cognitifs de base, in : Ch. Guimelli, (Ed.). Structures et transformations des représentations sociales, Neuchâtel : Delachaux et Nieslé, 171-198.

Guttman, L. (1950). The problem of attitude and opinion measurement. In : S.A Stouffer (Ed.) Measurement and prediction. Princeton : Princeton University Press, 60-90.

Hymes, D. (1962). The ethnography of speaking, In : T. Gladwin and W.C. Sturtevant (eds.), Anthropology and human behaviour, Washington, D.C. : Anthropological Society of Washington, $13-53$. 
Pour toute citation : références à rappeler

Salès-Wuillemin, E. (2006). Méthodologie de l'enquête, in : M., Bromberg et A., Trognon (Eds.) Psychologie Sociale 1, Presses Universitaires de France, 45-77.

Jakobi, J.M., Sales-Wuillemin, E., (1994), Les méthodes utilisant le langage, in : Ghiglione, R., Richard, J.F., (Eds.), Cours de Psychologie Tome 4 : Mesures et Analyses, Paris : Dunod, 171264.

Jodelet, D. (1986). Fous et folie dans un milieu rural français : une approche monographique. In W. Doise et A. Palmonari (Eds.), L'étude des représentations sociales. Neuchâtel : Delachaux et Niestlé, 171-192.

Jodelet, D., (Ed.) (1989), Les représentations sociales, Paris, Presses Universitaires de France ( $2^{\text {ème }}$ édition 1993).

Le Bouedec, G. (1984). Contribution à la méthodologie d'étude des représentations sociales, Cahiers de Psychologie Cognitive, 4, 3, 245-272.

Lebart, L., Salem, A. (1988). Analyse statistique des données textuelles. Paris : Dunod.

Likert, R., (1932). A technique for the measurement of attitudes. Archives of Psychology, 140, 166-189.

Matalon, B. (1992). Interrogations sur interrogation, Revue Internationale de psychologie Sociale, 5, 2, 99-114.

Moliner, P. (2001) (Ed.) La dynamique des représentations sociales, Grenoble : Presses Universitaires de Grenoble.

Moliner, P., Rateau, P., Cohen-Scali, V. (2002). Les représentations sociales : pratique des études de terrain, Grenoble : Presses Universitaires de Grenoble.

Monacci, C. (2002). La représentation groupale du bon manager, Mémoire de Master Professionnel de psychologie sociale du travail : ergologie relationnelle, Université de Bourgogne, sous la direction de E. Sales-Wuillemin, 250p.

Morin, M. (1999). Emergence du Sida et transformation des représentations sociales, in : M.L Rouquette et C. Garnier, La genèse des représentations sociales, Montréal : Editions Nouvelles, 14-41.

Moscovici, S., (1961), La psychanalyse son image et son public, Paris : Presses Universitaires de France (P.U.F)

Osgood, C.E., Suci, G.J., Tannenbaum, P.H. (1957). The Measurement of Meaning, Urbana, University of Illinois Press.

Pizzio, L., Lacassagne, M.-F., Sales-Wuillemin, E., (2003) La représentation du stage d'observation chez des étudiants de première année au travers de deux modes de recueil, Pratiques psychologiques, 1, 97-104. 
Pour toute citation : références à rappeler

Salès-Wuillemin, E. (2006). Méthodologie de l'enquête, in : M., Bromberg et A., Trognon (Eds.) Psychologie Sociale 1, Presses Universitaires de France, 45-77.

Rosenberg, M.J., Hovland, C.I., (1960), Cognitive, affective and behavioral components of attitudes, in : C.I., Hovland et M.J., Rosenberg (Eds.), Attitude Organization and Change, New Haven : Yale University Press.

Roulet, E., (1981). Echanges, Interventions et actes de langage dans la structure de la conversation, Etudes de Linguistique Appliquée, 44, 7-39.

Rouquette, M.-L., Rateau, P. (1998). Introduction à l'étude des représentations sociales, Grenoble : Presses Universitaires de Grenoble.

Sales-Wuillemin, E., Morlot, R., Dautun, M., Talon, D., Minary-Dohen, P. (2005). L'évolution d'une représentation sociale : l'exemple de l'hygiène chez une population spécialisée en soins infirmiers, Actes du Congrès National de la Société Française de Psychologie, Nancy : France, 21-23 Septembre 2005.

Sales-Wuillemin, E. (2005). Psychologie Sociale expérimentale de l'usage du langage. Représentations sociales, Catégorisation et attitudes : perspectives nouvelles, Paris : L'Harmattan, collection Figures de l'interaction

Sales-Wuillemin, E. (2006). La catégorisation et les stéréotypes en psychologie sociale, Paris : Dunod (Collection Psycho Sup).

Sales-Wuillemin, E., Bromberg, M. (2004). Modalités de constitution d'une représentation d'un objet nouveau, le cas des OGM, Actes de la sème conférence Internationale de l'ADRIPS, Lausanne, Suisse, 1er Septembre au 4 septembre 2004.

Sales-Wuillemin, E., Stewart, I. (2005). Locataire ou propriétaire : effet du statut des occupants sur la représentation de leur logement et sur les relations interindividuelles au sein de l'immeuble, Actes du 7ème Colloque International de Psychologie Sociale Appliquée, ADRIPS Rennes, France, 20- 22 Octobre 2005.

Searle, J.R., (1975), Indirect Speech Acts, in : P. Cole (Ed.), Syntax and Semantics, Vol. 3, Speech Acts, New-York : Academic Press.

Stewart, I., Sales-Wuillemin, E., Camara-Lima, L. (2005). Les effets de l'accession à la propriété sur la représentation du logement familial : perspectives psychosociale, in : M. BarréPépin, C. Coutant-Lapalus, (Eds). Logement et famille : des droits en question, Paris : Daloz. Thomas, W.I., Znaniecki, F., (1918-20), The polish peasant in Europe and America, Boston : Badger.

Wiener, N. (1948). Cybernetics, or Control and Communication in the Animal and the Machine, Paris : Hermann. 
Pour toute citation : références à rappeler

Salès-Wuillemin, E. (2006). Méthodologie de l'enquête, in : M., Bromberg et A., Trognon (Eds.) Psychologie Sociale 1, Presses Universitaires de France, 45-77.

Yzerbyt, V., Schadron, G. (1996). Connaître et juger autrui : Une introduction à la cognition sociale. Grenoble : Presses Universitaires de Grenoble.

Zanotti, J. (2002). La catégorisation sociale et les relations intergroupes à la Caisse d'Epargne de Bourgogne, Mémoire de Master Professionnel de psychologie sociale du travail : ergologie relationnelle, Université de Bourgogne, sous la direction de E. Sales-Wuillemin, 56p. 
Pour toute citation : références à rappeler

Salès-Wuillemin, E. (2006). Méthodologie de l'enquête, in : M., Bromberg et A., Trognon (Eds.) Psychologie Sociale 1, Presses Universitaires de France, 45-77.

\section{INDEX des concepts}

\begin{tabular}{|l|l|}
\hline Observation & \\
\hline Expérimentation & \\
\hline Attitudes & \\
\hline Observables & \\
\hline Composantes attitudinnelles & \\
\hline Préjugés/stéréotypes & \\
\hline Exostéréotype/endostéréotype & \\
\hline Question d'étude & \\
\hline Problématique & \\
\hline Variable Indépendante & \\
\hline Variable Dépendante & \\
\hline Hypothèses & \\
\hline Plan de recueil de données & \\
\hline Feed-back & \\
\hline Relances & \\
\hline Guide d'entretien & \\
\hline Distance sociale & \\
\hline Analyse lexicale & \\
\hline Lemmatisation & \\
\hline Analyse de similitude & \\
\hline Analyse thématique & \\
\hline Associations verbales & \\
\hline $\begin{array}{l}\text { Analyse Factorielle de Correspon- } \\
\text { dances }\end{array}$ & \\
\hline
\end{tabular}

\section{INDEX des auteurs}

\begin{tabular}{|l|l|}
\hline Eagly et Chaiken & \\
\hline Rosenberg et Hovland & \\
\hline Sales-Wuillemin & \\
\hline Jodelet & \\
\hline Abric & \\
\hline Flament & \\
\hline Rouquette et Guimelli & \\
\hline Ghiglione & \\
\hline Goffman & \\
\hline Blanchet & \\
\hline Matalon & \\
\hline Thurstone & \\
\hline Likert & \\
\hline Guttman & \\
\hline Osgood & \\
\hline Bogardus & \\
\hline
\end{tabular}


Pour toute citation : références à rappeler

Salès-Wuillemin, E. (2006). Méthodologie de l'enquête, in : M., Bromberg et A., Trognon (Eds.) Psychologie Sociale 1, Presses Universitaires de France, 45-77.

\section{PROPOSITION DE QUELQUES DEFINITIONS}

Attitude : Ensemble des connaissances, des réactions comportementales et affectives face à un objet attitudinal.

Attributions causales : processus consistant pour un sujet en la recherche des causes d'un événement. Il s'agit d'une auto attribution lorsque l'événement concerne le sujet qui réalise l'attribution causale, qu'il y est donc impliqué en tant qu'acteur, et d'hétéro attribution lorsque l'événement implique un autre individu, le sujet est donc concerné en tant qu'observateur. Deux ensembles de causes sont distingués : les causes internes (qui dépendent du sujet ou de l'individu impliqué dans l'événement) les causes externes (qui sont indépendantes).

Biais : source d'erreur non contrôlée.

Biais d'Endofavoritisme : biais qui consiste à favoriser les membres de son propre groupe social (endogroupe), en les jugeant de façon plus positive ou en leur attribuant un plus grand nombre de ressources.

Biais d'Exodéfavoritisme : biais qui consiste à défavoriser les membres des autres groupes sociaux (exogroupes), en les jugeant de façon plus négative ou en leur attribuant un moins grand nombre de ressources.

Biais de recueil : source d'erreur non contrôlée lors de la phase de recueil des données au sein d'un dispositif (biais de complaisance, d'estime de soi, de réactance, de conformisme, de valorisation de soi, défensif .....).

Catégorie : ensemble d'éléments regroupés parce qu'ils présentent une similarité c'est-à-dire qu'ils partagent un certain nombre de traits communs.

Contrat de communication : ensemble des paramètres que se fixent les interlocuteurs pour délimiter leurs productions discursives. Il prend en compte les statuts et rôles des interlocuteurs, leurs relations, leur histoire conversationnelle, l'objectif de la situation, le lieu dans lequel se déroule l'interaction, etc. (cf. Ghiglione 1986).

Enjeu : dans la théorie du contrat de communication, il s'agit de la force qui pousse un individu à entrer en communication, alors même que cela exigera de lui un effort cognitif et relationnel et que la situation peut présenter des risques en matière de perte de face. Selon Ghiglione (1986) il faut que l'enjeu soit suffisant pour que l'intra-locuteur accepte l'entrée en communication, dit autrement que le rapport coût/bénéfice attendu lui semble en sa faveur.

Préjugé : valeur positive ou négative associée à un ensemble social.

Représentation sociale : peut être considéré comme un processus ou un produit : processus consistant en la production et la prise en charge collective de l'image d'un objet social, ensemble de connaissances de sens commun partagé par un ensemble social et ayant une visée pratique (d'après Jodelet, 1989).

Stéréotypes sociaux : "croyances partagées au sujet des caractéristiques personnelles, généralement des traits de personnalité, mais aussi souvent des comportements, d'un groupe de personnes" (catégorielles, Yzerbyt, Schadron, 1996 : 24). On parle d'endostéréotype lorsque le stéréotype est appliqué au propre groupe social du sujet considéré (endogroupe), et d'exostéréotype lorsqu'il s'applique à un exogroupe. 